\title{
Archaic chaperone-usher pilus self-secretes into a superelastic zigzag spring architecture
}

\section{Anton Zavialov ( $\nabla$ antzav@utu.fi )}

University of Turku

\section{Natalia Pakharukova}

University of Turku https://orcid.org/0000-0002-8363-6105

Henri Malmi

University of Turku

Minna Tuittila

University of Turku

\section{Sari Paavilainen}

University of Turku

\section{Tobias Dahlberg}

Umeå University

\section{Magnus Andersson}

Umeå University https://orcid.org/0000-0002-9835-3263

\section{Si Lhyam Myint}

Umeå University

Bernt Eric Uhlin

Umeå University https://orcid.org/0000-0002-2991-8072

Debnath Ghosal

California Institute of Technology

\section{Yi-Wei Chang}

University of Pennsylvania https://orcid.org/0000-0003-2391-473X

\section{Grant Jensen}

California Institute of Technology https://orcid.org/0000-0003-1556-4864

\section{Stefan Knight}

Uppsala University https://orcid.org/0000-0002-7180-8758

Urpo Lamminmäki

University of Turku

\section{Biological Sciences - Article}

Keywords: Acinetobacter baumannii, archaic pili, ultrathin zigzag architecture 
Posted Date: November 5th, 2021

DOl: https://doi.org/10.21203/rs.3.rs-936177/v1

License: (c) (i) This work is licensed under a Creative Commons Attribution 4.0 International License. Read Full License

Version of Record: A version of this preprint was published at Nature on July 19th, 2022. See the published version at https://doi.org/10.1038/s41586-022-05095-0. 


\section{Archaic chaperone-usher pilus self-secretes into a superelastic}

\section{zigzag spring architecture}

Natalia Pakharukova ${ }^{1} \uparrow$, Henri Malmi $^{1} \uparrow$, Minna Tuittila ${ }^{1}$, Tobias Dahlberg ${ }^{2}$, Debnath Ghosal ${ }^{3}$, Yi-Wei Chang ${ }^{3}$, Si Lhyam Myint ${ }^{4}$, Sari Paavilainen ${ }^{1}$, Stefan David Knight ${ }^{5}$, Urpo Lamminmäki ${ }^{6}$, Bernt Eric Uhlin ${ }^{4}$, Magnus Andersson ${ }^{2}$, Grant Jensen ${ }^{3}$, Anton V. Zavialov ${ }^{1 *}$ Tykistökatu 6A, 20520, Turku, Finland.

${ }^{2}$ Department of Physics, Umeå Centre for Microbial Research (UCMR), Umeå University; Linnaeus väg 24, 90187 Umeå, Sweden.

${ }^{3}$ Division of Biology and Biological Engineering, California Institute of Technology; 1200 E California Blvd, Pasadena, CA91106, USA.

${ }^{4}$ Department of Molecular Biology and The Laboratory for Molecular Infection Medicine Sweden (MIMS), Umeå Centre for Microbial Research (UCMR), Umeå University; 6K och 6L, Sjukhusområdet, Umeå universitet, 90187 Umeå, Sweden.

${ }^{5}$ Department of Cell and Molecular Biology, Biomedical Centre, Uppsala University; Husargatan 3, 75237 Uppsala, Sweden.

${ }^{6}$ Department of Biochemistry, University of Turku; Tykistökatu 6A, 20520, Turku, Finland.

$\dagger$ These authors contributed equally

*Corresponding author. Email: anton.zavialov@utu.fi 


\section{Abstract}

Adhesive pili are hair-like appendages assembled via the chaperone-usher pathway (CUP) that mediate host tissue colonization and biofilm formation of Gram-negative bacteria ${ }^{1-3}$. Archaic CUP pili, the most diverse and widespread CUP adhesins, are promising vaccine and drug targets due to their prevalence in the most troublesome multidrug-resistant (MDR) pathogens 1,4,5. However, their architecture and assembly-secretion process remain unknown. Here, we present the $3.4 \AA$ resolution cryo-electron microscopy structure of the prototypical archaic Csu pilus that mediates biofilm formation of Acinetobacter baumannii, a notorious MDR nosocomial pathogen. In contrast to the thick helical tubes of the classical CUP pili, archaic pili assemble into a conceptually novel ultrathin zigzag architecture secured by an elegant clinch mechanism. The molecular clinch provides the pilus with high mechanical stability as well as superelasticity, a property observed now for the first time in biomolecules, while enabling a more economical and faster pilus production. Furthermore, we demonstrate that clinch formation at the cell surface drives pilus secretion through the outer membrane. These findings suggest that clinch-formation inhibitors might represent a new strategy to fight MDR bacterial infections.

\section{Main}

Adhesive pili are hair-like surface appendages that mediate bacterial infection and biofilm formation. In Gram-negative bacteria most adhesive pili are assembled from protein subunits via the classical, alternative, and archaic chaperone-usher pathways (CUPs) ${ }^{1}$. Whereas classical and alternative CUPs are restricted to $\beta$ - and $\gamma$-Proteobacteria, archaic CUPs are much more prevalent and present in a wide range of phyla ${ }^{1}$. Archaic CUPs are promising vaccine and drug targets due to their wide distribution in the most troublesome pathogens including panantibiotic-resistant Acinetobacter baumannii and Pseudomonas aeruginosa ${ }^{1,4,5}$. 
pathogen since it confers fitness for survival and persistence on surfaces ${ }^{4,6}$ and is mediated by Csu pili assembled via the archaic chaperone-usher CsuC-CsuD pathway ${ }^{4}$. The Csu pilus comprises the major subunit $\mathrm{CsuA} / \mathrm{B}$ that forms the pilus rod, adaptor subunits $\mathrm{CsuA}$ and $\mathrm{CsuB}$ and two-domain tip adhesin CsuE that binds to various substrates using exposed hydrophobic finger-like loops ${ }^{7}$. In contrast to thick and rigid fibres of classical and alternative CUPs, Csu pili are surprisingly thin ${ }^{7}$, suggesting a drastically different pilus architecture. Classical pili form quaternary structures by packing into a thick, hollow helical tube ${ }^{8-10}$ that can elongate and unwind to resist strong rinsing flows ${ }^{11}$. The molecular architecture and biomechanical properties of archaic pili are unknown. Thus, we sought to obtain a structure of the Csu pilus rod by cryo-electron microscopy (cryo-EM).

\section{Archaic CUP pili are stiff ultrathin zigzag filaments secured by clinch mechanism}

Cryo-EM micrographs revealed thin and long, but remarkably stiff pili, much resembling animal hairs (Fig. 1a). The structure of the Csu pilus rod was determined to an overall resolution of $3.4 \AA$ (Extended Data Fig. 1 and Table 1, Supplementary Video 1). The Csu pilus P pili made of the same number of subunits (Fig. 1d). Hence, the archaic assembly appears is a thin $(\sim 23 \AA)$ left-handed filament with a helical rise $(z)$ of $28.0 \AA$ and rotation between subunits $(\varphi)$ of $-153^{\circ}$ (Fig. $1 \mathrm{~b}, \mathrm{c}$ ). Pilins are tilted $\sim 60^{\circ}$ relative to the helical axis and $\sim 69^{\circ}$ relative to each other, resulting in a zigzag appearance. This architecture is strikingly different from that of classical pili (Fig. 1d) ${ }^{9}$. Remarkably, Csu rods are three times longer than rods of not by one, but two binding mechanisms. First, the incoming CsuA/ $\mathrm{B}^{\mathrm{N}+1}$ pilin subunit inserts its donor strand $\mathrm{Gd}^{\mathrm{N}+1}$ into the hydrophobic groove of the preceding $\mathrm{CsuA} / \mathrm{B}^{\mathrm{N}}$ subunit (Fig. more economical and Csu pili are expected to grow in length three times faster than P pili at the same rate of pilin incorporation.

In contrast to their classical counterparts, the subunits in Csu rods are linked together 2a). This is similar to donor strand complementation (DSC) in other CUPs ${ }^{12-16}$. The second 
binding mechanism, unique to archaic systems, involves a $\mathrm{A}^{\prime}-\mathrm{A}^{\prime \prime}, \mathrm{B}-\mathrm{B}^{\prime}$ twin hairpin, which protrudes like an arm from one side of CsuA/B (Fig. 2a and Extended Data Fig. 2). In the pilus, $\mathrm{Csu} A / \mathrm{B}^{\mathrm{N}+1}$ not only provides $\mathrm{Gd}^{\mathrm{N}+1}$ to $\mathrm{CsuA} / \mathrm{B}^{\mathrm{N}}$, but also inserts the $\mathrm{A}^{\prime}-\mathrm{A}^{\prime \prime}$ hairpin into a pocket in CsuA/B $\mathrm{B}^{\mathrm{N}}$ between $\beta$ strands $\mathrm{B}$ and $\mathrm{E} 2$ and loop D-D' (Fig. 2a, b). In addition, the $\mathrm{Gd}^{\mathrm{N}+2}$ of the third subunit $\mathrm{CsuA} / \mathrm{B}^{\mathrm{N}+2}$ that complements $\mathrm{CsuA} / \mathrm{B}^{\mathrm{N}+1}$ binds with its protruding $\mathrm{N}$-terminal part to $\beta$ strands $\mathrm{A}^{\prime \prime}$ and $\mathrm{B}$ in $\mathrm{CsuA} / \mathrm{B}^{\mathrm{N}}$. Thereby, $\mathrm{CsuA} / \mathrm{B}^{\mathrm{N}}$ becomes firmly clinched between two extended surfaces of the CsuA/ $\mathrm{B}^{\mathrm{N}+1}-\mathrm{Gd}^{\mathrm{N}+2}$ module (Fig. 2a-c): one from the $\mathrm{A}^{\prime}-$ $\mathrm{A}^{\prime \prime}$ hairpin (Fig. 2c, magenta) and the other from the N-terminal part of $\mathrm{Gd}^{\mathrm{N}+2}$ (Fig. 2c, orange). Finally, residues in $\beta$ strand $\mathrm{A}$ and loop $\mathrm{A}-\mathrm{A}^{\prime}$ in $\mathrm{CsuA} / \mathrm{B}^{\mathrm{N}+1}$ form several contacts with residues in the $\mathrm{A}^{\prime \prime}-\mathrm{B}$ loop at the 'bottom' of $\mathrm{CsuA} / \mathrm{B}^{\mathrm{N}}$, thereby bridging the two main binding sites to form a continuous binding surface of $600 \AA^{2}$ with 41 interacting residues and 13 hydrogen bonds (Fig. 2b, Supplementary Video 1). Altogether, the clinch contact provides nearly onethird of the total interactive surface (32\%) and hydrogen bond network (31\%) between pilins.

The structure of the clinch contact somewhat resembles the arrangement of vertebrate jaws (Fig. 2c, Supplementary Video 2). The linker between Gd and the globular domain of a

\section{Archaic CUP pili act as superelastic zigzag springs}

The biomechanical properties of a single Csu pilus were probed by force spectroscopy using optical tweezers (OT) (Fig. 3a). The force response under extension (black curve) comprises three regions previously reported for classical P pili ${ }^{17,18}$. Initially, the force increases linearly with extension, representing an elastic stretching of the rod (region I). Then the force is constant 
quaternary helical conformation of the rod. Hence, in Csu pili, it should correspond to a linearization of the zigzag filament. Finally, the force increases again linearly and shifts to a sigmoidal shape representing elastic stretching and a conformational change of the pilins (region III) ${ }^{18}$. When reversing the movement and allowing the pilus to rewind, classical and archaic pili show a dramatically different contraction response. Classical pili exhibit a dip in force associated with slack in the pilus needed to restore the helical structure ${ }^{19}$. In contrast, in the Csu pilus the contraction response perfectly tracks that of the extension, similarly to shape memory metals that regain the original shape after deformation by external stress. Therefore, the archaic Csu pilus acts like a superelastic molecular zigzag spring. persistence length, suggesting a straight structure and high stiffness (Fig. 3b). Remarkably, the mean length of region II is identical to the estimated length of an average pilus (Fig. 3a, b). Thus, the Csu zigzag filament reversibly unwinds to a linear conformation that is exactly twice its length. This almost integer elongation ratio (2.01) is also revealed by modelling and is the result of the peculiar geometry of the pilus: opening the clinch changes the tilt of the pilin from $\sim 60^{\circ}$ to $\sim 0^{\circ}$ or from cosine $\sim 0.5$ to $\sim 1$ (Fig. $3 \mathrm{c}$ ). The Csu zigzag filament is less extendible than helical tubes like P pili, which can unwind to 5-7 times their original length ${ }^{20}$. However, Csu pili can be stretched significantly further at higher forces due to a conformational change in pilins (region III).

The average force required to open the clinch contact is similar to unwinding forces for many tightly packed helical tube pili (Fig. 3) ${ }^{20}$. This remarkable coincidence suggests that the two different architectures both evolved to adapt to similar shear forces. However, Csu pili are more dynamic than their classical counterparts. Whereas the unwinding force for classical P pili rapidly increases at velocities above $0.4 \mu \mathrm{m} / \mathrm{s}^{17}$, the force response of Csu pili remains the same at velocities up to $20 \mu \mathrm{m} / \mathrm{s}$. The rapid response of the Csu zigzag filament to the extension force is likely due to their linearized quaternary structure, in which subunits have only contacts 
with the nearest neighbours. In contrast, in helical tubes of $\mathrm{P}$ pili each pilin interacts with ten other subunits, which greatly reduces the unwinding rate, restricting the ability to respond to sudden changes or fluctuations in fluid flow rate ${ }^{21}$. Thus, archaic zigzag filaments can potentially mediate bacterial attachment in turbulent environments.

\section{Quaternary structure formation drives pilus secretion}

Considering the large interactive area of the clinch, we hypothesized that the clinch or its formation might have additional functions, and assessed its role in pilus stability, assembly and secretion by mutagenesis. First, we replaced a large portion of the $\mathrm{A}^{\prime}-\mathrm{A}^{\prime \prime}$ hairpin (residues TEGNMN) by a single glycine ( $\Delta 6$, Extended Data Tables $2-3)$. The $\Delta 6$ CsuA/Bsc mutant showed similar levels of expression and thermal stability as wild-type (WT) CsuA/Bsc (Fig. 4a, Extended Data Fig.6 and Table 3). Moreover, the deletion had no effect on the usher-free assembly of CsuA/B polymers in the periplasm (Fig. 4b). However, the $\Delta 6$ mutation completely abolished pilus expression on the cell surface suggesting that the deletion disrupted pilus translocation through the usher channel (Fig. 4c, Extended Data Fig. 3a, b). Similarly, shorter and larger deletions within the $\mathrm{A}^{\prime}-\mathrm{A}^{\prime \prime}$ hairpin did not affect subunit stability or conformation of the $\mathrm{A}^{\prime}-\mathrm{A}^{\prime \prime}$ hairpin.

Surprisingly, the deletions within the $\mathrm{A}^{\prime}-\mathrm{A}^{\prime \prime}$ and the B-B' hairpins did not prevent biofilm formation (Fig. 4d), though the mutations made biofilms more susceptible to the inhibition with anti-tip (anti-CsuE N-terminal domain) antibody. Consistent with this finding, we detected the CsuE tip subunit on the surface of the mutant bacteria using $\mathrm{Eu}^{3+}$ labelled antitip antibody (Extended Data Fig.4). This result suggests that some form of short pili or tip 
fibrilium, composed of subunits CsuA, CsuB, and CsuE, may still assemble and present the tip-fingers adhesion site on the cell surface.

We analysed the effects of point mutations in the clinch interface (Extended Data Figs 3-6 and Table 3). Substitutions in key residues of the $\mathrm{A}^{\prime}-\mathrm{A}^{\prime \prime}$ hairpin and acceptor site, except for partially buried Met27, abolished or severely inhibited secretion, permitting assembly of only a few pilus-like structures on some bacteria. To probe the role of the Gd N-terminus in clinch formation, we substituted Val2 mediating an important hydrophobic contact between the interacting pilins with alanine (Fig. 2b). The mutation was well tolerated and did not affect assembly, but resulted in atypical, apparently short pili (Extended Data Fig. 3 and Table 3). much shorter in all three extension regions (Fig. 3a). The seven times shorter unwinding region II suggests that Val2Ala pili are seven times shorter than WT pili (Fig. 3b). Furthermore, the opening of the mutation-impaired clinch contacts required a much lower tensile force. Therefore, this mutation provides an intermediate case suggesting that the length of secreted pili correlates positively with the strength of the clinch contact. Taken together, clinch formation is coupled to pilus rod secretion and is necessary for efficient expression of Csu pili on the cell surface.

To understand how clinch formation may facilitate secretion, we modelled the assembly-secretion process based on the available structures from the Csu system and classical CUPs (Fig. 4e, Extended Data Fig.7 and Supplementary Video 3). The incoming CsuC-CsuA/B preassembly complex ${ }^{22}$ is recruited to the usher N-terminal domain (NTD) whereas the chaperone-capped base of the growing pilus (represented by Sub1-Sub2-Sub3 fragment) is positioned at the usher C-terminal domains CTD1 and CTD2 ${ }^{23-26}$ (steps 1-2, Fig. 4e and Extended Data Fig.7). The Gd of chaperone-bound Sub4 replaces the $\mathrm{G}_{1}$ strand of the base chaperone through donor strand exchange (DSE), linking Sub4 to Sub3 ${ }^{14,15}$. DSE results in the complete folding of Sub3 and formation of the $\mathrm{A}^{\prime}-\mathrm{A}^{\prime \prime}$ and B-B' hairpins ${ }^{27}$. However, Sub3 
cannot form a clinch contact with its neighbouring subunits, as Sub2 has entered the narrow usher channel and the chaperone-bound Sub4 is only partially folded and lacks its own twinhairpin ${ }^{22}$. The clinch contact can only be formed between subunits Sub1 and Sub2 on the cell surface (Fig. 4e, step 3). Therefore, the formation of the twin hairpin not prior to, but after DSE serves three purposes. First, it provides an additional folding potential to drive assembly ${ }^{27}$. Second, it prevents premature subunit clinching, thereby keeping the fibre in the elongated conformation required for secretion. Finally, it enables the formation of the quaternary structure immediately after subunit translocation.

The secretion step involves handover of the base from NTD to CTDs. The handover cannot be driven by the binding of the base to CTDs, as neither significant hydrophobic interactions nor affinity between the base and CTDs have been observed (Extended Data Fig.8), questioning the origin of forces and energy driving secretion in CUPs. The FimD usher NTD was recently shown to escort the base until it reaches CTDs and form interactions with CTD2 that could potentially facilitate the release of the base from NTD ${ }^{28}$. Our findings demonstrate that secretion of the pilus rod is greatly facilitated by quaternary structure formation, representing an alternative driving force. Since formation of a single clinch reduces the fibre length exactly by the length of one pilin, clinch formation may actively pull subunits to the cell surface without introducing shifts in their positions at each cycle (Extended Data Fig.7). In addition, clinch formation may prevent backtracking of the secretion step potentially leading to the base slipping away from the usher after its release from NTD, permanently jamming assembly (Extended Data Fig.9). Future structural studies on the assembly-secretion

\section{Discussion}

24 The clinch-DSC-based zigzag filament is probably the earliest and the most widely used architecture of pili assembled via the CUP. This economical design gives the pilus a 
surprisingly high mechanical stability, rapid dynamic properties and superelasticity. The pilus secretion process involves an elegant mechanism that allows clinch formation only at the cell surface (Fig. 4e). Hence, similar to the chaperone that preserves folding energy of the subunit to drive pilus assembly, the usher inhibits the formation of the quaternary structure, preserving energy of inter-subunit contacts to drive pilus secretion through the membrane. Interestingly, polymers of classical CUP subunits can easily adopt the zigzag filament architecture of the archaic pili (Extended Data Fig.10), suggesting that both types of pili might follow a similar conserved secretion pathway before they reach the stage of forming the final quaternary bacterial infections.

\section{References}

1 Nuccio, S. P. \& Baumler, A. J. Evolution of the chaperone/usher assembly pathway: fimbrial classification goes Greek. Microbiol Mol Biol Rev 71, 551-575, (2007).

2 Zav'yalov, V., Zavialov, A., Zav'yalova, G. \& Korpela, T. Adhesive organelles of Gramnegative pathogens assembled with the classical chaperone/usher machinery: structure and function from a clinical standpoint. FEMS Microbiol Rev 34, 317-378, (2010).

3 Busch, A. \& Waksman, G. Chaperone-usher pathways: diversity and pilus assembly mechanism. Philosophical transactions of the Royal Society of London. Series B, Biological sciences 367, 1112-1122, (2012).

4 Tomaras, A. P., Dorsey, C. W., Edelmann, R. E. \& Actis, L. A. Attachment to and biofilm formation on abiotic surfaces by Acinetobacter baumannii: involvement of a novel chaperone-usher pili assembly system. Microbiology 149, 3473-3484, (2003). 
5 Giraud, C., Bernard, C. S., Calderon, V., Yang, L., Filloux, A., Molin, S., Fichant, G., Bordi, C. \& de Bentzmann, S. The PprA-PprB two-component system activates CupE, the first non-archetypal Pseudomonas aeruginosa chaperone-usher pathway system assembling fimbriae. Environmental microbiology 13, 666-683, (2011).

6 Cerqueira, G. M. \& Peleg, A. Y. Insights into Acinetobacter baumannii pathogenicity. IUBMB Life 63, 1055-1060, (2011).

7 Pakharukova, N., Tuittila, M., Paavilainen, S., Malmi, H., Parilova, O., Teneberg, S., Knight, S. D. \& Zavialov, A. V. Structural basis for Acinetobacter baumannii biofilm

8 Mortezaei, N., Epler, C. R., Shao, P. P., Shirdel, M., Singh, B., McVeigh, A., Uhlin, B. E., Savarino, S. J., Andersson, M. \& Bullitt, E. Structure and function of enterotoxigenic Escherichia coli fimbriae from differing assembly pathways. Mol Microbiol 95, 116-126, (2015).

9 Hospenthal, M. K., Redzej, A., Dodson, K., Ukleja, M., Frenz, B., Rodrigues, C., Hultgren, S. J., DiMaio, F., Egelman, E. H. \& Waksman, G. Structure of a Chaperone-Usher Pilus Reveals the Molecular Basis of Rod Uncoiling. Cell 164, 269-278, (2016).

10 Hospenthal, M. K., Zyla, D., Costa, T. R. D., Redzej, A., Giese, C., Lillington, J., Glockshuber, R. \& Waksman, G. The Cryoelectron Microscopy Structure of the Type 1 Chaperone-Usher Pilus Rod. Structure 25, 1829-1838 e1824, (2017).

11 Barbercheck, C. R. E., Bullitt, E. \& Andersson, M. Bacterial Adhesion Pili. Subcell Biochem 87, 1-18, (2018).

12 Choudhury, D., Thompson, A., Stojanoff, V., Langermann, S., Pinkner, J., Hultgren, S. L. \& S. D. Knight. X-ray structure of the FimC-FimH chaperone-adhesin complex from uropathogenic Escherichia coli. Science 285, 1061-1066, (1999). 
13 Sauer, F. G., Futterer, K., Pinkner, J. S., Dodson, K. W., Hultgren, S. J. \& Waksman, G. Structural basis of chaperone function and pilus biogenesis. Science 285, 1058-1061, (1999).

14 Zavialov, A. V., Berglund, J., Pudney, A. F., Fooks, L. J., Ibrahim, T. M., MacIntyre, S. \& Knight, S. D. Structure and biogenesis of the capsular F1 antigen from Yersinia pestis: preserved folding energy drives fiber formation. Cell 113, 587-596, (2003).

15 Sauer, F. G., Pinkner, J. S., Waksman, G. \& Hultgren, S. J. Chaperone priming of pilus subunits facilitates a topological transition that drives fiber formation. Cell 111, 543-551, (2002).

16 Remaut, H., Rose, R. J., Hannan, T. J., Hultgren, S. J., Radford, S. E., Ashcroft, A. E. \& Waksman, G. Donor-strand exchange in chaperone-assisted pilus assembly proceeds through a concerted beta strand displacement mechanism. Mol. Cell 22, 831-842, (2006).

17 Andersson, M., Fallman, E., Uhlin, B. E. \& Axner, O. Dynamic force spectroscopy of E. coli P pili. Biophys J 91, 2717-2725, (2006).

18 Andersson, M., Fallman, E., Uhlin, B. E. \& Axner, O. A sticky chain model of the elongation and unfolding of Escherichia coli P pili under stress. Biophys J 90, 1521-1534, (2006).

19 Andersson, M., Axner, O., Almqvist, F., Uhlin, B. E. \& Fallman, E. Physical properties of biopolymers assessed by optical tweezers: analysis of folding and refolding of bacterial pili. Chemphyschem 9, 221-235, (2008).

20 Jass, J., Schedin, S., Fallman, E., Ohlsson, J., Nilsson, U. J., Uhlin, B. E. \& Axner, O. Physical properties of Escherichia coli P pili measured by optical tweezers. Biophys $J$ 87, 4271-4283, (2004).

21 Andersson, M., Uhlin, B. E. \& Fallman, E. The biomechanical properties of E. coli pili for urinary tract attachment reflect the host environment. Biophys $J$ 93, 3008-3014, (2007). 
22 Pakharukova, N., Garnett, J. A., Tuittila, M., Paavilainen, S., Diallo, M., Xu, Y., Matthews, S. J. \& Zavialov, A. V. Structural Insight into Archaic and Alternative Chaperone-Usher Pathways Reveals a Novel Mechanism of Pilus Biogenesis. PLoS Pathog 11, e1005269, (2015).

23 Nishiyama, M., Horst, R., Eidam, O., Herrmann, T., Ignatov, O., Vetsch, M., Bettendorff, P., Jelesarov, I., Grutter, M. G., Wuthrich, K., Glockshuber, R. \& Capitani, G. Structural basis of chaperone-subunit complex recognition by the type 1 pilus assembly platform FimD. EMBO J. 24, 2075-2086, (2005).

24 Phan, G., Remaut, H., Wang, T., Allen, W. J., Pirker, K. F., Lebedev, A., Henderson, N. S., Geibel, S., Volkan, E., Yan, J., Kunze, M. B., Pinkner, J. S. et al. Crystal structure of the FimD usher bound to its cognate FimC-FimH substrate. Nature 474, 49-53, (2011).

25 Yu, X., Dubnovitsky, A., Pudney, A. F., Macintyre, S., Knight, S. D. \& Zavialov, A. V. Allosteric Mechanism Controls Traffic in the Chaperone/Usher Pathway. Structure 20, 1861-1871, (2012).

26 Geibel, S., Procko, E., Hultgren, S. J., Baker, D. \& Waksman, G. Structural and energetic basis of folded-protein transport by the FimD usher. Nature 496, 243-247, (2013).

27 Pakharukova, N., McKenna, S., Tuittila, M., Paavilainen, S., Malmi, H., Xu, Y., Parilova, O., Matthews, S. \& Zavialov, A. V. Archaic and alternative chaperones preserve pilin folding energy by providing incomplete structural information. J Biol Chem 293, 1707017080, (2018).

28 Du, M., Yuan, Z., Yu, H., Henderson, N., Sarowar, S., Zhao, G., Werneburg, G. T., Thanassi, D. G. \& Li, H. Handover mechanism of the growing pilus by the bacterial outermembrane usher FimD. Nature 562, 444-447, (2018).

29 Zheng, S. Q., Palovcak, E., Armache, J. P., Verba, K. A., Cheng, Y. \& Agard, D. A. MotionCor2: anisotropic correction of beam-induced motion for improved cryo-electron microscopy. Nat Methods 14, 331-332, (2017). 
$30 \mathrm{He}, \mathrm{S}$. \& Scheres, S. H. W. Helical reconstruction in RELION. Journal of structural biology 198, 163-176, (2017).

31 Tang, G., Peng, L., Baldwin, P. R., Mann, D. S., Jiang, W., Rees, I. \& Ludtke, S. J. EMAN2: an extensible image processing suite for electron microscopy. Journal of structural biology 157, 38-46, (2007).

32 Desfosses, A., Ciuffa, R., Gutsche, I. \& Sachse, C. SPRING - an image processing package for single-particle based helical reconstruction from electron cryomicrographs. Journal of structural biology 185, 15-26, (2014).

33 Emsley, P., Lohkamp, B., Scott, W. G. \& Cowtan, K. Features and development of Coot. Acta Crystallogr D Biol Crystallogr 66, 486-501, (2010).

34 Adams, P. D., Grosse-Kunstleve, R. W., Hung, L. W., Ioerger, T. R., McCoy, A. J., Moriarty, N. W., Read, R. J., Sacchettini, J. C., Sauter, N. K. \& Terwilliger, T. C. PHENIX: building new software for automated crystallographic structure determination. Acta. Crystallogr. D Biol. Crystallogr. 58, 1948-1954, (2002).

35 Chen, V. B., Arendall, W. B., 3rd, Headd, J. J., Keedy, D. A., Immormino, R. M., Kapral, G. J., Murray, L. W., Richardson, J. S. \& Richardson, D. C. MolProbity: all-atom structure validation for macromolecular crystallography. Acta Crystallogr D Biol Crystallogr 66, 12-21, (2010).

36 Balsalobre, C., Morschhauser, J., Jass, J., Hacker, J. \& Uhlin, B. E. Transcriptional analysis of the sfa determinant revealing mmRNA processing events in the biogenesis of S fimbriae in pathogenic Escherichia coli. J Bacteriol 185, 620-629, (2003).

37 Stangner, T., Dahlberg, T., Svenmarker, P., Zakrisson, J., Wiklund, K., Oddershede, L. B. \& Andersson, M. Cooke-Triplet tweezers: more compact, robust, and efficient optical tweezers. Optics Letters 43, 1990-1993, (2018).

38 Andersson, M., Czerwinski, F. \& Oddershede, L. B. Optimizing active and passive calibration of optical tweezers. J Optics-Uk 13, (2011). 
39 Kelley, L. A., Mezulis, S., Yates, C. M., Wass, M. N. \& Sternberg, M. J. The Phyre2 web portal for protein modeling, prediction and analysis. Nature protocols 10, 845-858, (2015).

40 Nishiyama, M., Vetsch, M., Puorger, C., Jelesarov, I. \& Glockshuber, R. Identification and characterization of the chaperone-subunit complex-binding domain from the type 1 pilus assembly platform FimD. J. Mol. Biol. 330, 513-525, (2003).

41 Dubnovitsky, A. P., Duck, Z., Kersley, J. E., Hard, T., MacIntyre, S. \& Knight, S. D.

\section{Methods}

\section{Bacterial strains and plasmids}

Escherichia coli strain DH5a was used for plasmid propagation. Protein expression was done in E. coli BL21-AI (Invitrogen). Expression plasmids were constructed based on the pBAD$\mathrm{Csu}^{7}$, pET101-6HCsuA/Bdsc ${ }^{27}$, and pET101-CsuC6H-CsuA/B ${ }^{22}$. Deletions and substitutions were generated using reverse PCR. The oligonucleotides are listed in Extended Data Table 2.

\section{Protein expression and purification}

Wild type (WT) and mutant CsuA/B were co-expressed with the CsuC chaperone, carrying a C-terminal His6-tag, in the periplasm of E. coli harbouring the pET101-CsuC6H-CsuA/B-\#\# plasmid series and were co-purified by $\mathrm{Ni}$-chelate chromatography essentially as described in ${ }^{22}$. WT and mutant CsuA/Bsc were present in the periplasm of $E$. coli harbouring the pET101$6 \mathrm{HCsuA} / \mathrm{Bdsc}$-\#\# plasmid series and were purified by $\mathrm{Ni}$-chelate chromatography as described earlier ${ }^{27}$. Depending on the remaining impurities, proteins were dialyzed against $20 \mathrm{mM}$ bis- 
TRIS propane, $\mathrm{pH} 9.0$ and were purified further by anion exchange chromatography on a Mono Q 5/50 GL column (GE Healthcare). For the circular dichroism measurements, the buffer for the proteins was exchanged to $12.5 \mathrm{mM}$ potassium phosphate, $\mathrm{pH} 7.0$ using a $\mathrm{PD}-10$ desalting column (GE Healthcare). Protein concentrations were measured on a NanoDrop ${ }^{\mathrm{TM}} 2000$ Spectrophotometer (Thermo Scientific).

To express wild-type and mutant variants of Csu fimbriae, E.coli BL-21 AI cells were transformed with ampicillin-resistant $\mathrm{pBAD}-\mathrm{Csu}$ and its derivatives. Selected clones were cultivated in Luria-Bertani (LB) medium supplemented with $100 \mu \mathrm{g} \mathrm{ml}^{-1}$ ampicillin overnight

\section{Cryo-electron microscopy} using a Vivaspin device (Sartorius Stedim) with a molecular mass cut-off of $100 \mathrm{kDa} .4 \mu \mathrm{l}$ of 
sample was applied to glow-discharged Quantifoil R2/2 300 mesh copper grids coated with ultrathin carbon (Electron Microscopy Sciences). The grids were blotted and plunge-frozen in liquid ethane using Vitrobot Mark IV (ThermoFisher Scientific) at $4{ }^{\circ} \mathrm{C}$ and $100 \%$ humidity. The data were collected on a $300 \mathrm{kV}$ Titan Krios electron microscope (Thermo Scientific) equipped with a Gatan K3 direct electron detector operated in super-resolution mode with a pixel size of $0.433 \AA$ and a defocus range of -1.0 to $-3.0 \mu \mathrm{m}$. A total dose of 60 electrons $/ \AA$ was applied and equally divided among 40 frames to allow for dose weighting. Details on cryoEM data collection are summarized in Extended Data Table 1.

\section{Cryo-EM image processing and reconstruction}

Dose-fractionated movie frames were subjected to beam-induced motion correction using MotionCor2 ${ }^{29}$. Image processing and helical reconstruction were performed in RELION 3.0 within EMAN $2{ }^{31}$ were subjected to $2 \mathrm{D}$ classification to generate auto-picking templates. After autopicking of helical filaments, a total of 480,064 segments were extracted with a box size of 400 pixels. After 2D and 3D classification steps 255,833 segments were used for 3D refinement. The segments were rescaled to a pixel size of $1.35 \AA$. A starting model for reconstruction was generated de novo from the 2D particles using Stochastic Gradient Descent algorithm in RELION 3.0. The helical symmetry parameters were estimated using conventional Fourier-Bessel analysis and segclassreconstruct and seggridexplore modules in SPRING ${ }^{32}$. The initial estimates of helical parameters ( $-157^{\circ}$ helical twist, $26.3 \AA$ helical rise) were tested using a search range of $-150^{\circ}$ to $-165^{\circ}$ for the twist and $26 \AA$ to $30 \AA$ for the rise. After $3 \mathrm{D}$ classification 255,833 particles were used for high resolution 3D refinement. The helical symmetry $\left(-153^{\circ}\right.$ helical twist, $28 \AA$ helical rise) was applied and refined during high resolution 3D refinement producing a map with a resolution of $6.18 \AA$. Applying a soft mask with a raised cosine edge of 14 pixels and B-factor sharpening yielded a map with a global resolution of 4.8 
$1 \AA$ assessed by the gold standard Fourier-shell correlation procedure between independently refined half reconstructions (FSC 0.143) (Scheres and Chen, 2012). The resolution was further improved to $3.42 \AA$ after two iterations of Bayesian polishing followed by 3D refinement and post-processing. The final map showed clear $\beta$-strand separation and density for bulky side chains consistent with the reported resolution. The pixel size of the cryo-EM maps from RELION was slightly off and was adjusted by calculating the cross-correlation coefficient of the map to the refined model using "Fit in Map" tool in UCSF Chimera package (Pettersen et al., 2004).

\section{Model building and refinement}

The initial model of the Csu pilus was built manually by fitting the crystal structure of the UCSF Chimera. The angle between two subunits was adjusted using the Chimera "Fit in Map" tool in several iterations of first docking three subunit dimers into adjacent regions in the map with one subunit overlap and averaging the orientations of the overlapping subunits, then overlapping the three dimers fully and averaging the subunit orientations of all three dimers. A short linker connecting the donor strand with strand A was modelled with Coot $^{33}$. The structure was refined by combining manual adjustments in Coot and real space refinement in PHENIX 34. The initial four-subunit model was reduced to a model with three donor strand complemented subunits (four chains) that occupy the highest resolution positions in the map. The model was validated with MolProbity ${ }^{35}$. The refinement statistics are given in Extended Data Table 1.

\section{Atomic force microscopy}

The bacteria were grown on the LB agar plate supplemented with carbenicillin and induced with $0.02 \%$ arabinose to produce pili. The bacterial cells with pili were imaged by atomic force microscopy (AFM) as described earlier ${ }^{36}$ with some modifications. Briefly, bacterial cells were 
suspended in $100 \mu \mathrm{L}$ of Milli-Q water and $10 \mu \mathrm{L}$ of which was placed onto a freshly cleaved mica surface (Goodfellow Cambridge Ltd., Cambridge). Samples were incubated for 5 min at room temperature and blotted dry before they were placed into a desiccator for a minimum of $2 \mathrm{~h}$ in order to dry. Images were collected with a Nanoscope V Multimode 8 AFM equipment (Bruker) using Bruker ScanAsyst mode with Bruker ScanAsyst-air probe oscillated at resonant frequency of $50-90 \mathrm{kHz}$, selected by the Nanoscope software. Images were collected in air at a scan rate of $0.8-1.5 \mathrm{~Hz}$, depending on the size of the scan and the number of samples $(256$ or 512 samples/image). The final images were plane fitted in both axes and presented in amplitude (error) mode.

\section{Biofilm assay and biofilm inhibition}

E. coli strain BL21 harboring pBAD-Csu or its derivatives was cultured overnight in Luria Broth (LB) medium in the presence of $100 \mathrm{mg}^{-1}$ ampicillin. $5 \mathrm{ml}$ of the fresh medium in a 50 $\mathrm{ml}$ polypropylene tube was inoculated with $100 \mu \mathrm{l}$ of the overnight culture and then grown at $37^{\circ} \mathrm{C}$ with vigorous shaking for $2 \mathrm{~h}$. Dilutions of the anti-CsuE $\mathrm{E}_{\mathrm{N}}\left(\alpha \mathrm{E}_{\mathrm{N}}\right)$ polyclonal antibody (Innovagen $\mathrm{AB}$ ) in $50 \mu \mathrm{LB}$ were divided on microtiter plates. Bacterial cultures were induced with $0.2 \%$ arabinose, and $150 \mu$ triplicates were mixed with the serum dilutions on microtiter plates. The plate was incubated at $37^{\circ} \mathrm{C}$ for $2 \mathrm{~h}$ with gentle shaking. Wells were then emptied and washed two times with $300 \mu 1$ of phosphate buffered saline. Any remaining biofilm was stained with $1 \%$ crystal violet for 15 min, rinsed with water, allowed dry, and dissolved in 250

\section{Western blotting}

Periplasmic fractions were mixed with Laemmli buffer, and the samples were boiled. The proteins were separated by electrophoresis in $18 \%$ SDS polyacrylamide gels and transferred onto an Immuno-blot polyvinylidene difluoride membrane (Bio-Rad Laboratories, USA) in 
Bio-Rad A-buffer (25 mM Tris, pH 8.3, $192 \mathrm{mM}$ glycine, with 20\% methanol and 0.1\% SDS) at $100 \mathrm{~V}$ or $350 \mathrm{~mA}$ for $1 \mathrm{~h}$. Membrane was blocked with 5\% skim milk in phosphate-buffered saline/Tween, incubated with primary anti-CsuA/B rabbit polyclonal antibody (Innovagen AB) followed by incubation with secondary IRDye 680RD-conjugated anti-rabbit goat antibody (Li-Cor Biosciences). Protein bands were detected with the Odyssey system (Li-Cor Biosciences) and quantified with ImageJ.

\section{Force measuring optical tweezers}

To measure the biomechanical properties of Csu pili we used an custom made force measuring optical tweezers setup constructed around an inverted microscope Olympus IX71 (Olympus, Japan) equipped with a water immersion objective (model: UPlanSApo60XWIR 60X N.A. = 1.2; Olympus, Japan) and a 1920 x 1440 pixel CMOS camera (model: C11440-10C, Hamamatsu) ${ }^{37}$. To sample force data with high signal-to-noise ratio with minimal amount of drifts we used the Allan variance method to identify noise ${ }^{38}$. We used the Power Spectrum method to calibrate the trap by sampling the microspheres position at $131,072 \mathrm{~Hz}$ and averaging 32 consecutive data sets acquired for $0.25 \mathrm{~s}$ each. To extend a pilus, we moved the piezo stage at a constant speed of $50 \mathrm{~nm} / \mathrm{s}$ and sampled the force and position at $50 \mathrm{~Hz}$. To assess the mean contour length of the pilus quaternary structure, we "buckled" pili by reversing the piezo-stage until the bead touched the bacterial cell wall. We measured the distance from our starting position (rise of region I) to the cell. To study ultrashort pili, we had to "rub" the bacterial surface with the laser-controlled bead in our OT system.

\section{Temperature-depended folding transition analysis}

Circular dichroism was measured using Chirascan ${ }^{\mathrm{TM}}$ CD Spectrometer (Applied Photophysics) and a Macro-cuvette 110-QS with $1 \mathrm{~mm}$ layer thickness (Hellma). Background for the spectra was first measured four times from the buffer $12.5 \mathrm{mM}$ potassium phosphate at $\mathrm{pH} 7.0$ before inserting the target protein at $0.150 \mathrm{mg} / \mathrm{ml}$ concentration. $\mathrm{CD}$ spectra at $20^{\circ} \mathrm{C}$ were measured 
four times with a 195-260 nm wavelength range and using $1 \mathrm{~nm}$ intervals between each $3 \mathrm{~s}$ measurements. For the melting spectra, proteins were heated using $4^{\circ} \mathrm{C}$ temperature ramping from 19 to $99^{\circ} \mathrm{C}$. Each spectrum was measured once after a $30 \mathrm{~s}$ temperature stabilization time using wavelength range $260-195 \mathrm{~nm}$ and $1 \mathrm{~nm}$ intervals between each $2 \mathrm{~s}$ measurements. The measurement of all melting spectra took $1 \mathrm{~h} 28 \mathrm{~min}$. Each spectrum was smoothed by a factor of 4. Melting curves were recorded at $225 \mathrm{~nm}$ wavelength by heating samples from 20 to $99^{\circ} \mathrm{C}$ at the rate of $1{ }^{\circ} \mathrm{C} / \mathrm{min}$. Circular dichroism was measured for $12 \mathrm{~s}$ at first every $1.0^{\circ} \mathrm{C}$ and later every $0.5^{\circ} \mathrm{C}$ with $0.15^{\circ} \mathrm{C}$ error margin. Each recording took $1 \mathrm{~h} 19 \mathrm{~min}$. The cuvette was purified of residual protein using $2 \mathrm{M}$ potassium hydroxide between samples. The "Curve Fitting function" in the Chirascan user interface was used to fit melting data to the "Sigmoid

\section{Modeling of assembly-secretion process}

The Csu pilus models were constructed based on the cryo-EM structure of the Csu pilus rod (this article) and the crystal structures of CsuA/Bsc (PDB: 6FM5) ${ }^{27}$ and CsuC-CsuA/B chaperone-subunit complex (PDB: 5D6H) ${ }^{22}$. With no structure for the CsuD usher available, the models of the usher were based on the structures of the FimD usher from the classical CU pathway: the crystal structure (PDB: 3RFZ) ${ }^{24}$ and cryo-EM structures of conformers 1 and 2 (PDB codes 6E14 and 6E15, respectively, ${ }^{28}$. The Phyre2 protein fold recognition server ${ }^{39}$ automatically modelled $92 \%$ of the full CsuD amino acid sequence based on the structure of the entire FimD (conformer 1) with a confidence value of $100.0 \%$. Models of the N-terminal domain (NTD) of the usher at different steps of pilus secretion were produced based on the crystal structures of NTD bound to preassembly complexes (PDB codes 1ZE3 and 4B0M) ${ }^{23}$ 25 and cryo-EM structures of the FimD conformers. Clashes between proteins were prevented between CsuA/B subunits and kept to the minimum elsewhere. Stereochemistry was analysed with COOT. 


\section{Data availability}

The coordinates were deposited to the Protein Data Bank with the accession code 7B78. The corresponding cryo-EM map was deposited in the EMDB with accession code EMD-12080.

Acknowledgments We thank the staff of the Cryo-EM Swedish National Facility at SciLifeLab, Stockholm and the Beckman Institute Resource Center for Transmission Electron Microscopy at Caltech, Pasadena for their assistance during data collection. We are grateful to Prof. Carsten Sachse, Prof. Juha Huiskonen and Stefan Huber for the helpful suggestions on helical.

Author contributions N.P., H.M., M.T., T.D., D.G., Y.W.C., S.L.M., S.P. and A.V.Z. performed experiments, AVZ, GJ, MA and BEU supervised the work, A.V.Z., N.P., G.J., M.A., B.E.U., U.L. and S.D.K. analysed the data and wrote the paper.

Funding This work was supported by grants from the Academy of Finland (321762) and S. Juselius Foundation (2019) to A.V.Z., National Institutes of Health (RO1 AI127401) to G.J., the Swedish Research Council (SRC) (2019-04016) to M.A., SRC (2019-01720) and The Kempe Foundations (JCK-1724) to B.E.U and SRC (2016-04451) to S.D.K.

Competing interests Authors declare that they have no competing interests. 

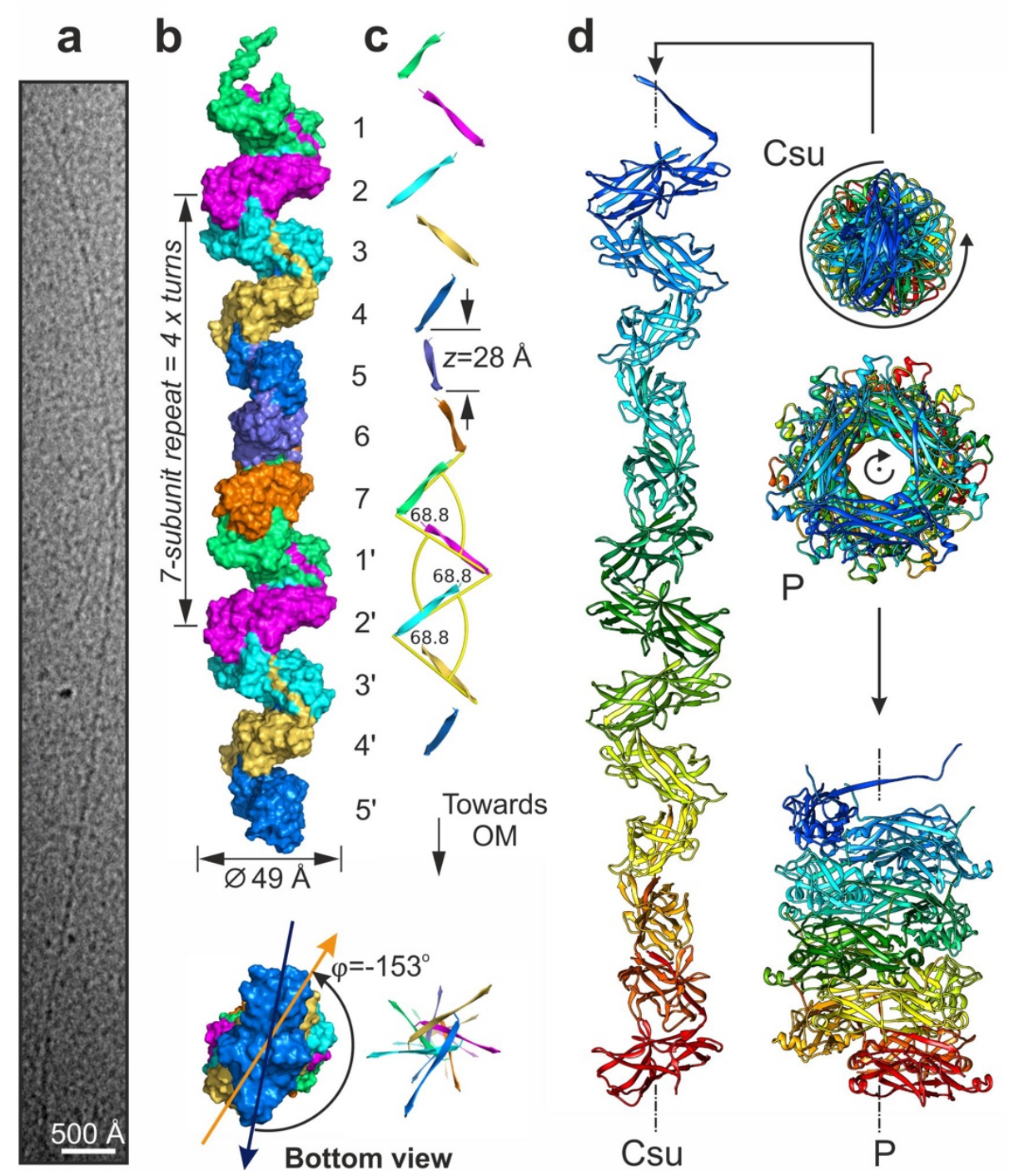

Fig. 1 | Csu pilus rod is a thin zigzag-like filament. a, Cryo-EM image of Csu pilus. b,

4 Surface diagram of a 12-subunt fragment of the Csu pilus rod. Subunits are numbered in the 5 direction of pilus growth, from the pilus tip to its assembly base at the outer membrane (OM).

6 c, Cartoon diagram of the rod focusing on the donor strands. d, Cartoon diagrams of 13-subunit fragments of archaic Csu and classical P pilus rods. The zigzag filament is $\sim 3$ times as long as the helical tube rod. The handedness is indicated by a black curved arrow. 

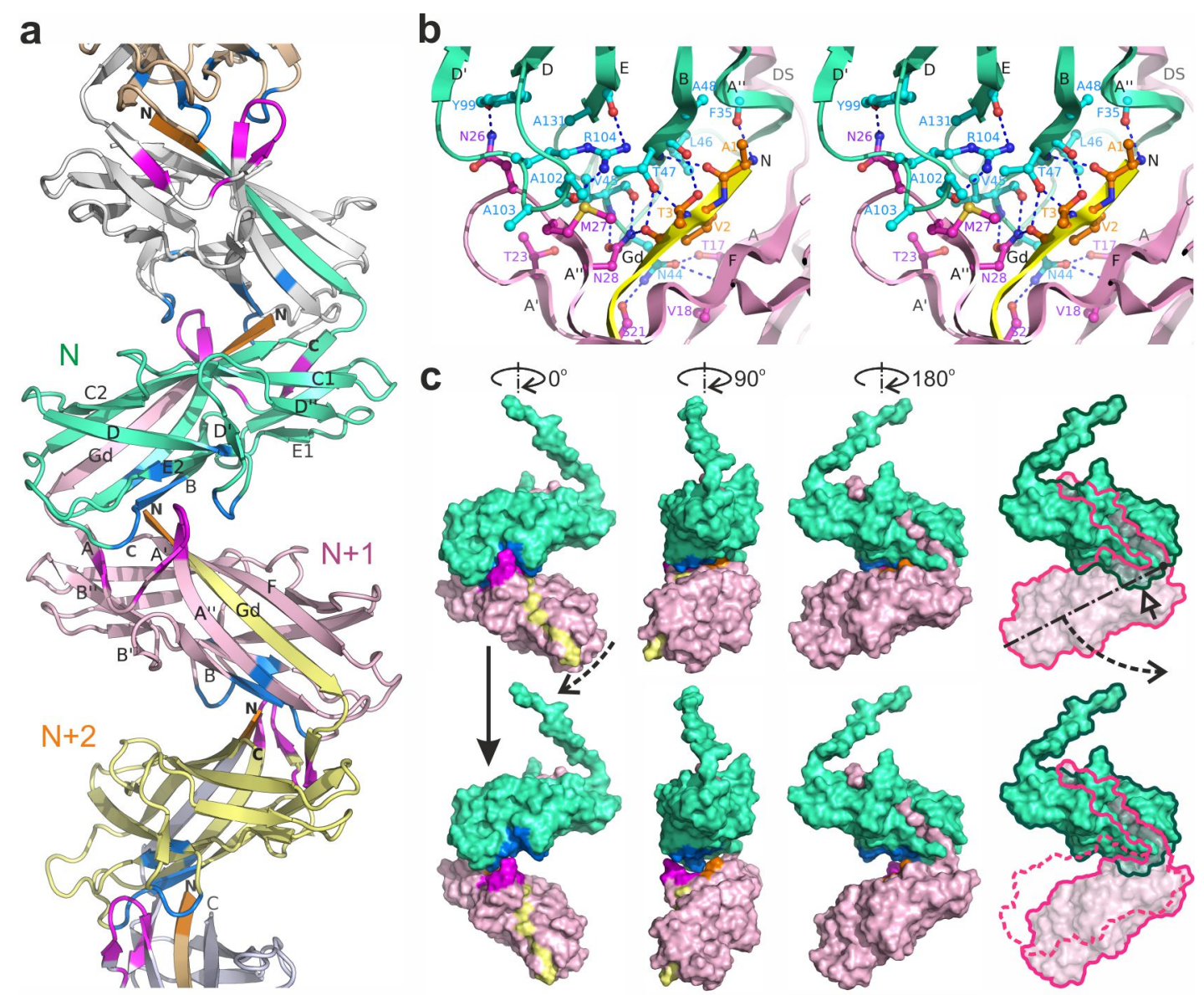

Fig. 2 | Csu pilus subunits are assembled by donor strand complementation and clinch

mechanisms. a, Cartoon diagram of the rod. Clinch contact residues in the A strand and A'$\mathrm{A}^{\prime \prime}$ hairpin, Gd donor strand N-terminus, and acceptor site are painted magenta, orange, and marine, respectively. $\mathrm{N}$ - and $\mathrm{C}$-termini as well as $\beta$-strands in the two central subunits are labelled. b, Clinch contact (stereo view). Adjacent subunits $\mathrm{CsuA} / \mathrm{B}_{\mathrm{N}}$ and $\mathrm{CsuA} / \mathrm{B}_{\mathrm{N}+1}$, and $\mathrm{Gd}_{\mathrm{N}+2}$ of subunit $\mathrm{CsuA} / \mathrm{B}_{\mathrm{N}+2}$ complementing $\mathrm{CsuA} / \mathrm{B}_{\mathrm{N}+1}$, are painted green, pink, and yellow, respectively. Interacting residues are shown as balls and sticks. Dashed lines represent hydrogen bonds. See also Supplementary Video 1. c, The jaw-like structure of the clinch determines pilus rigidity and trajectory of subunit movement upon clinch formation or pilus stretching. Molecular surface of two adjacent subunits (green and pink) and Gd of the third subunit (yellow) is shown in three orientations obtained by viewing the structure after rotation around the pilus helical axis as indicated. Residues involved in the clinch are painted as in $\mathbf{a}$.

13 A model of a partially opened conformation produced by rotating the lower subunit by $\sim 17^{\circ}$ 14 around the linker is shown below. The open arrowhead shows surface buried between subunits. 15 See also Supplementary Video 2. 
a
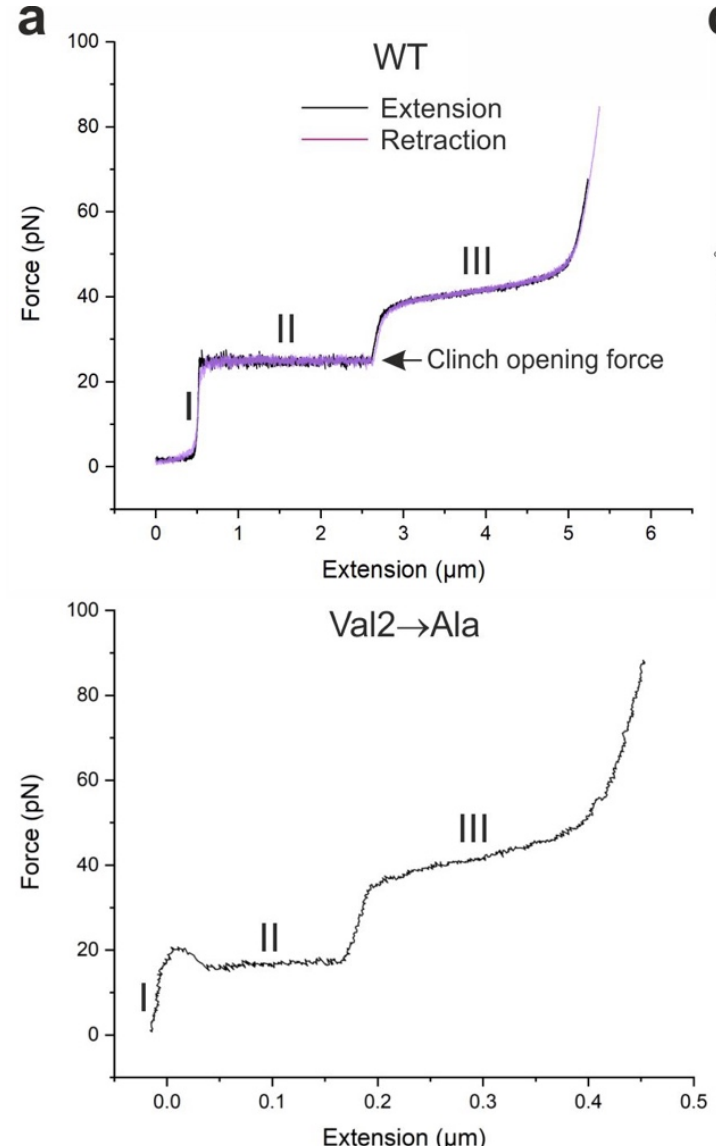

C

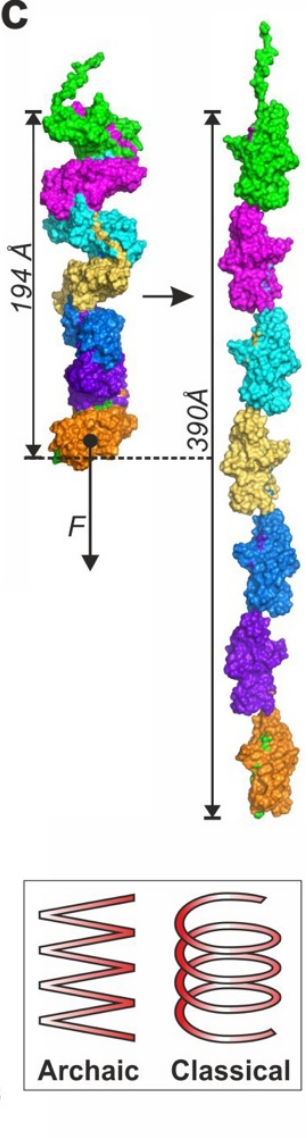

b

\begin{tabular}{lll}
\hline \multicolumn{1}{c}{ Property } & \multicolumn{1}{c}{ WT } & \multicolumn{1}{c}{ Val2Ala } \\
\hline Pilus contour length, $\mu \mathrm{m}$ & $1.7 \pm 0.6, \mathrm{n}=10$ & $\mathrm{NE}$ \\
Pilus persistence length, $\mu \mathrm{m}$ & $0.89 \pm 0.17, \mathrm{n}=16$ & $\mathrm{NE}$ \\
Unwinding region II, $\mu \mathrm{m}$ & $1.7 \pm 0.2, \mathrm{n}=36$ & $0.24 \pm 0.05, \mathrm{n}=18$ \\
"S" region III, $\mu \mathrm{m}$ & $2.1 \pm 0.6, \mathrm{n}=10$ & $0.36 \pm 0.10, \mathrm{n}=8$ \\
Clinch opening force, $\mathrm{pN}$ & $23 \pm 0.2, \mathrm{n}=41$ & $15 \pm 0.4, \mathrm{n}=22$ \\
\hline * Not estimated (NE) because of the short length of Val2Ala pili
\end{tabular}

Fig. 3 | Archaic pilus rod is a molecular zigzag spring that exhibits superelastic properties.

a, Force-extension and retraction measurement of a single Csu pilus using optical tweezers. $\mathbf{b}$, Measurements. c, Csu pilus can be stretched to twice its length. Surface diagrams of a 7-subunt 4 helical repeat of the rod and a model of the maximally extended fibre. Insert: Conceptual 5 difference in the molecular spring design between archaic (zigzag) and classical (helical) pilus 6 rods. 

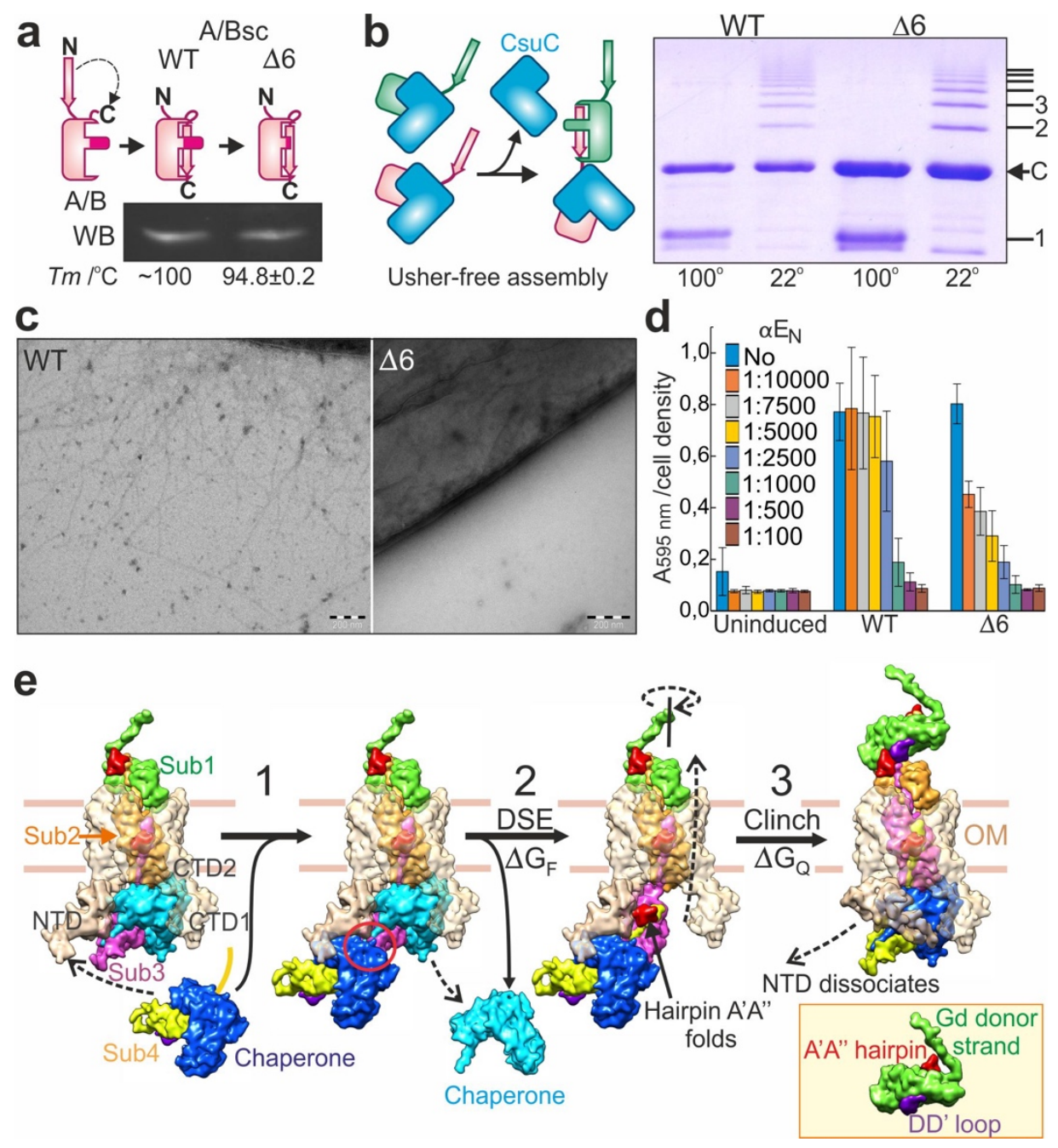

Fig. 4 | Clinch contact is required for efficient secretion of the pilus rod to the cell surface.

a, Schematic of wild type (WT) and $\Delta 6 \mathrm{CsuA} / \mathrm{Bsc}$ constructs and western blot (WB) analysis of the periplasm extracted from E. coli expressing these constructs. Melting temperature of purified proteins is indicated below (see also Extended Data Fig.6 and Table 3). b, Schematic of CsuC-assisted assembly of CsuA/B and SDS-PAGE of CsuC-(CsuA/B $)_{n}$ complexes purified from the periplasm of cells co-expressing CsuC with WT or $\Delta 6 \mathrm{CsuA} / \mathrm{B}$ and pre-incubated at 22 or $100^{\circ} \mathrm{C}$. Positions of $\mathrm{CsuC}$ and $(\mathrm{CsuA} / \mathrm{B})_{n}$ with $\mathrm{n}=1-7$ are indicated. $\mathbf{c}$, Negative-stained TEM micrographs of E. coli harbouring WT or $\Delta 6 c s u$ gene cluster. d, Biofilm formation by WT or $\Delta 6$ csu E. coli at different concentrations of anti-tip antibody $\alpha \mathrm{E}_{\mathrm{N}}$. Analysis of other mutants is shown in Extended Data Figs. 3-6 and the data is summarized in Extended Data Table 3. e, Csu pilus assembly-secretion cycle. Modelling is described in Extended Data Fig.7. 2 The $\Delta \mathrm{G}_{\mathrm{F}}$ folding energy and $\Delta \mathrm{G}_{\mathrm{Q}}$ free energy of quaternary structure formation preserved by $14 \quad$ Video 3. 

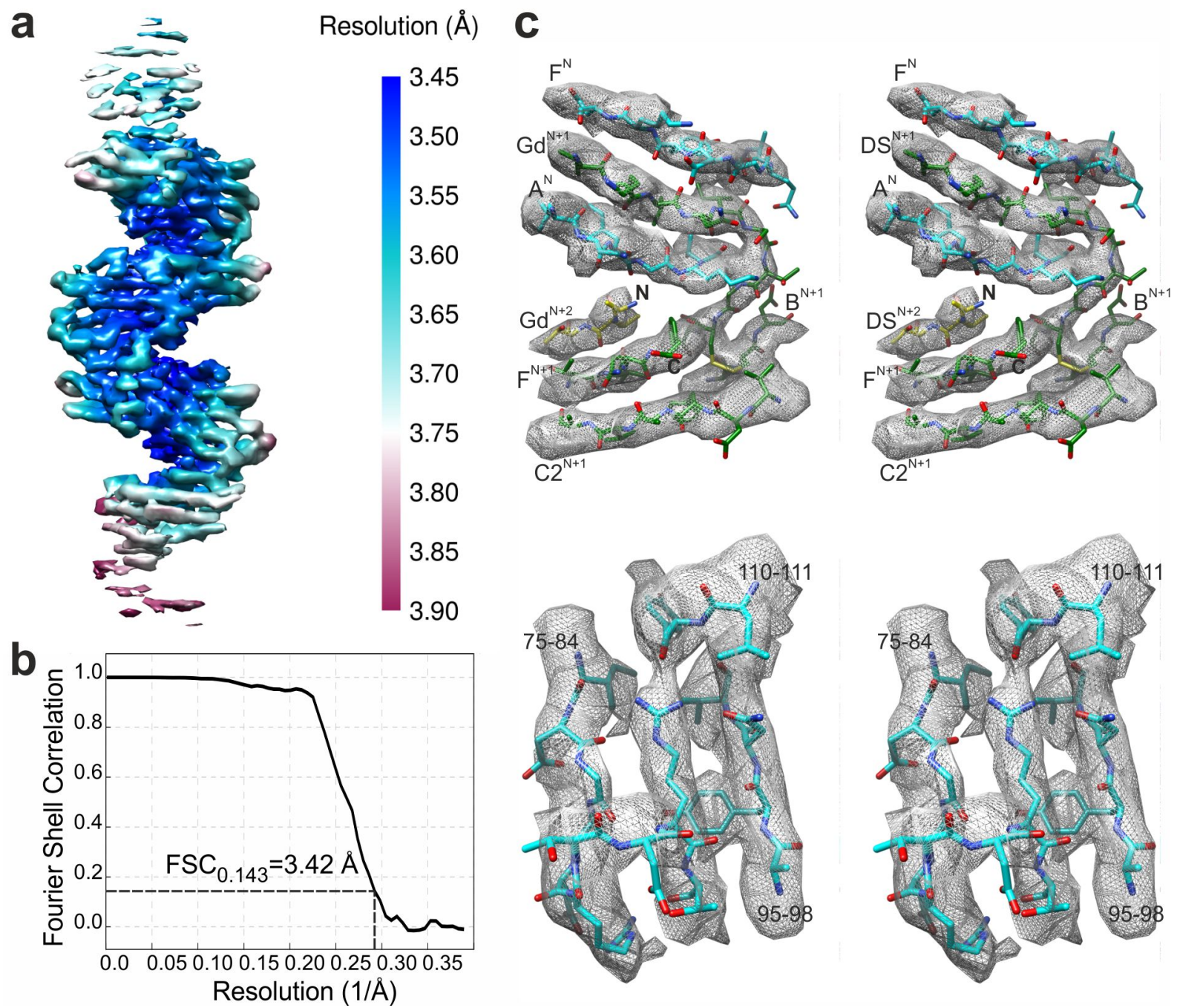

Extended Data Fig. 1 | Resolution estimation and selected regions of the 3D electron microscopy map of the Csu pilus rod. a, Local-resolution estimation. b, The Gold-standard Fourier shell correlation estimation at the 0.143 correlation threshold. c, Model fitting in density map (stereo view). Upper panel: Density for the interactive area between adjacent subunits $C s u A / B^{N}$ and $C s u A / B^{N+1}$, and $G d$ of subunit $C s u A / B^{N+2}\left(G d^{N+2}\right)$. Strands $A^{N}$ and $F^{N}$ in $\operatorname{CsuA} / \mathrm{B}^{\mathrm{N}}$, strands $\mathrm{B}^{\mathrm{N}+1}, \mathrm{C} 2^{\mathrm{N}+1}$, and $\mathrm{F}^{\mathrm{N}+1}$ in $\mathrm{CsuA} / \mathrm{B}^{\mathrm{N}+1}$ and donor strands $\mathrm{Gd}^{\mathrm{N}+1}$ and $\mathrm{Gd}^{\mathrm{N}+2}$ are labelled. Low panel: Density for a fragment of the subunit core, including residues 75-84, 95-98, and 110-111. 

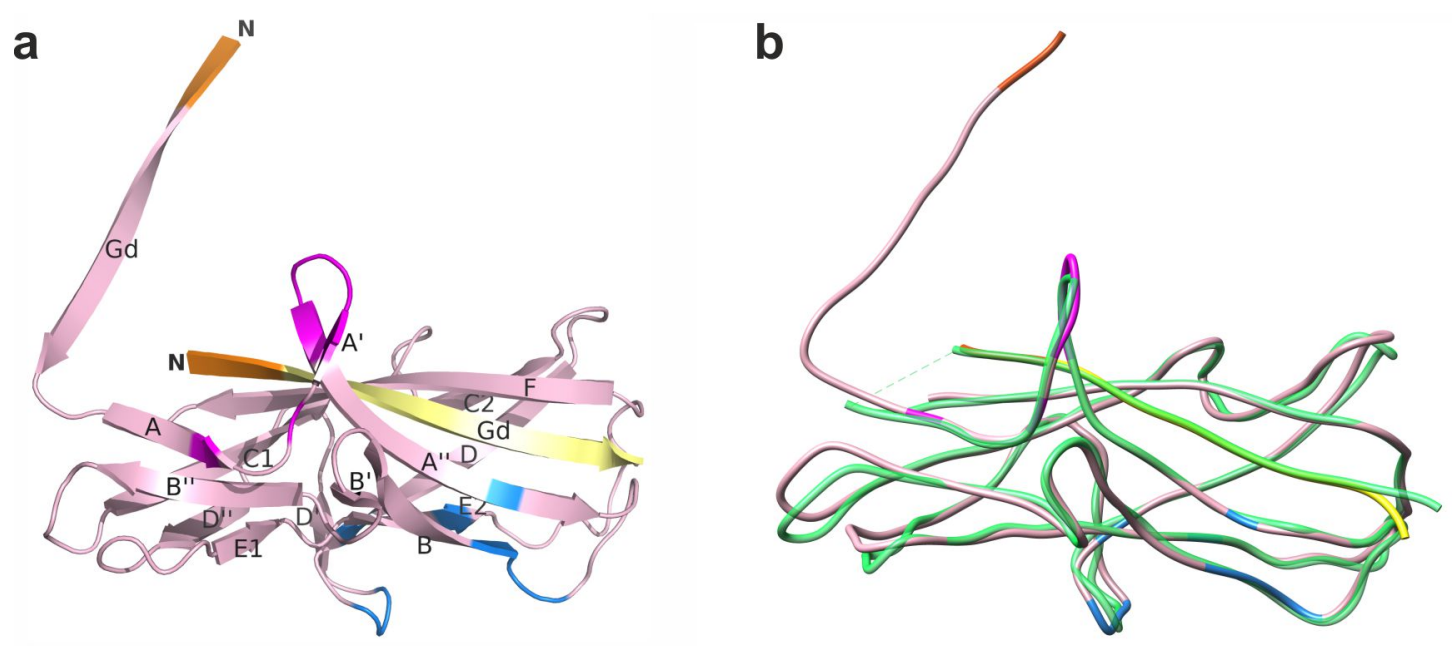

Extended Data Fig. 2 | Structure of the CsuA/B subunit in the pilus rod. a, Cartoon representation of $\mathrm{CsuA} / \mathrm{B}^{\mathrm{N}}$ (pink) complemented by the $\mathrm{Gd}$ donor strand of the next subunit $\mathrm{CsuA} / \mathrm{B}^{\mathrm{N}+1}$ (yellow) in the rod. Clinch contact residues in the A strand and $\mathrm{A}^{\prime}-\mathrm{A}^{\prime \prime}$ hairpin, the $\mathrm{N}$-terminus of donor strand $\mathrm{Gd}$, and the acceptor site are painted magenta, orange, and marine, respectively. N-termini and $\beta$-strands are labelled. $\mathbf{b}$, Superposition of the pilus $\mathrm{CsuA} / \mathrm{B}^{\mathrm{N}}$ $\mathrm{Gd}^{\mathrm{N}+1}$ module with the crystal structure of self-complemented CsuA/B (CsuA/Bsc, PDB code 6FM5, Pakharukova et al. 2018, ribbon diagram). CsuA/ $\mathrm{B}^{\mathrm{N}}-\mathrm{Gd}^{\mathrm{N}+1}$ is painted as in a and $\mathrm{CsuA} / \mathrm{Bsc}$ is painted green. The dashed line indicates unstructured linker in CsuA/Bsc that connects the $\mathrm{Gd}$ strand to the $\mathrm{C}$-terminus. 


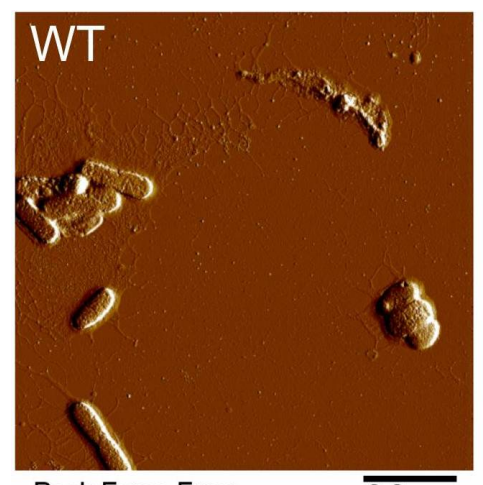

Peak Force Error

$\overline{3.0 \mu \mathrm{m}}$
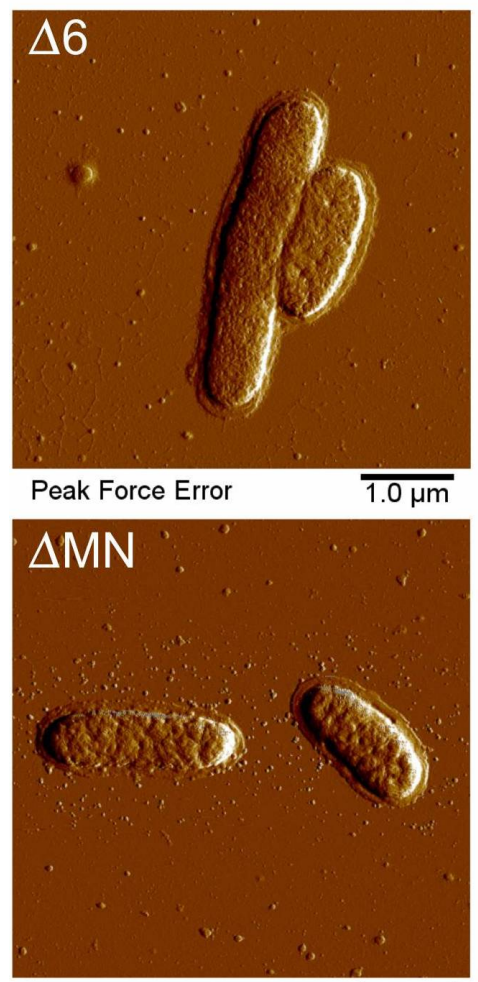

Peak Force Error $\overline{1.0 \mu \mathrm{m}}$

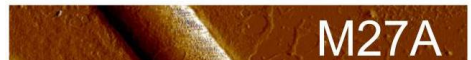

in

Peak Force Error

$\overline{1.0 \mu \mathrm{m}}$

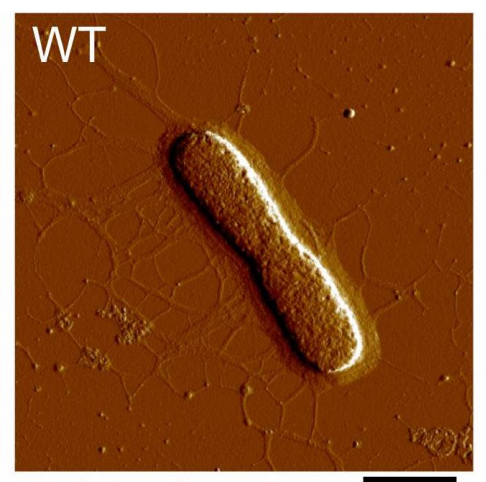

Peak Force Error
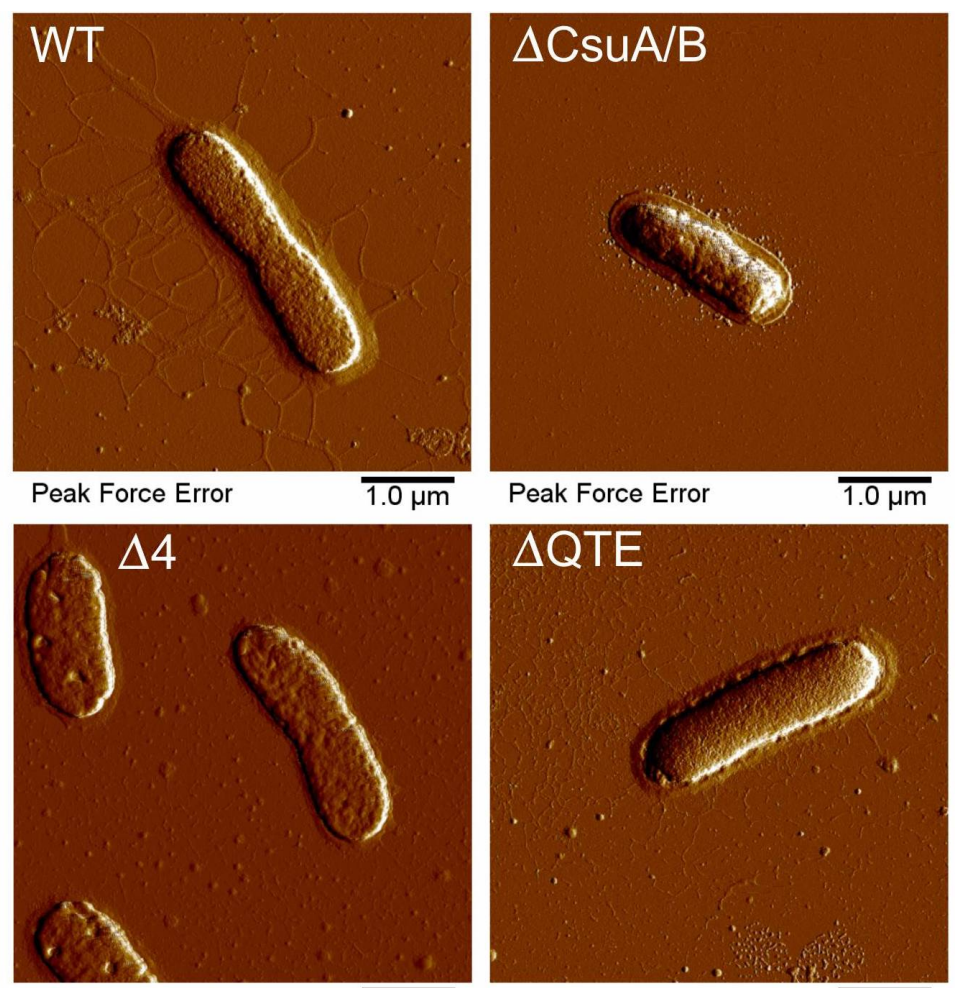

$\triangle$ QTE
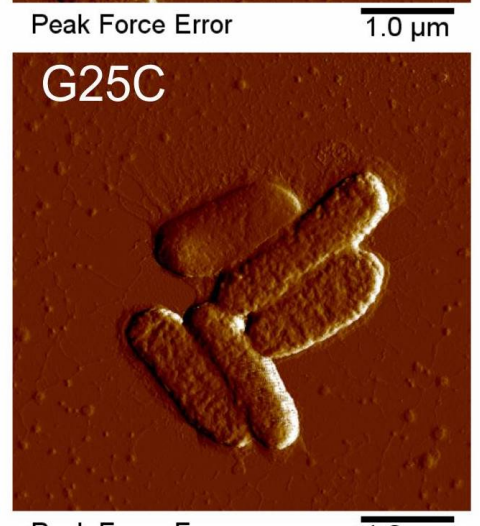

Peak Force Error

$\overline{1.0 \mu \mathrm{m}}$
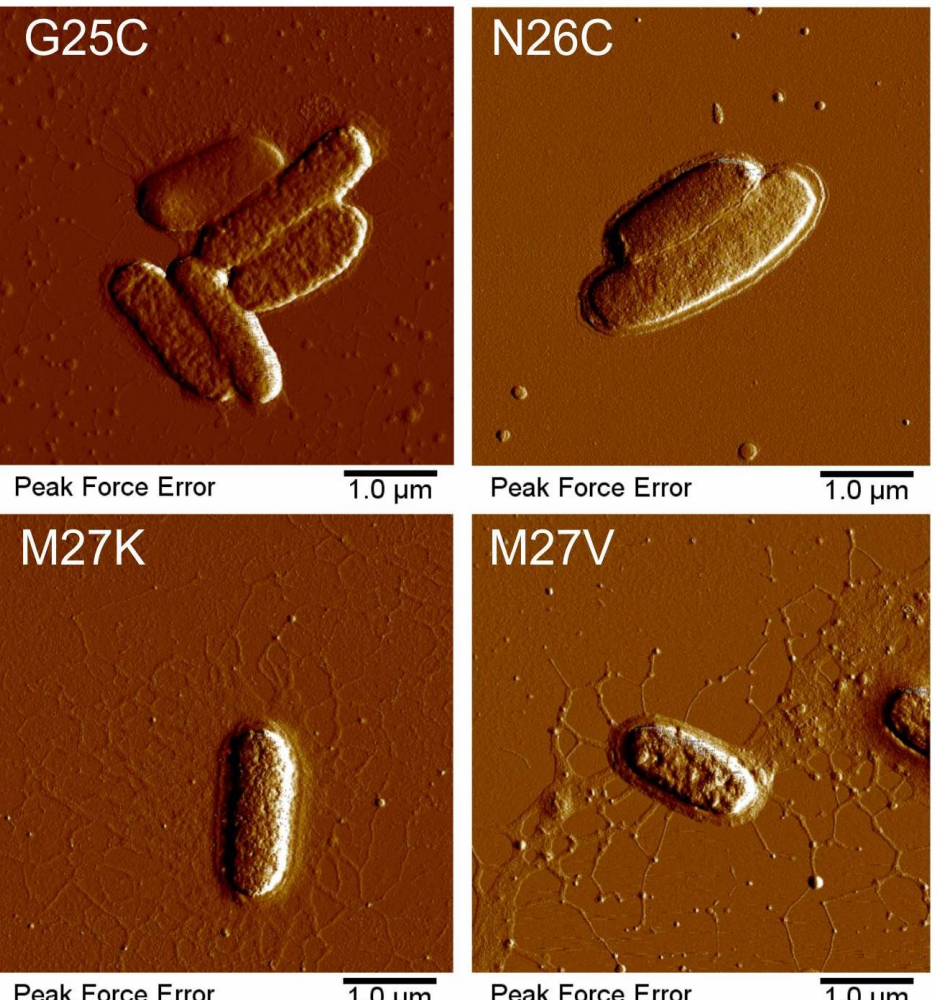

Peak Force Error

Peak Force Error

$\overline{1.0 \mu \mathrm{m}}$

Continues on the next page 

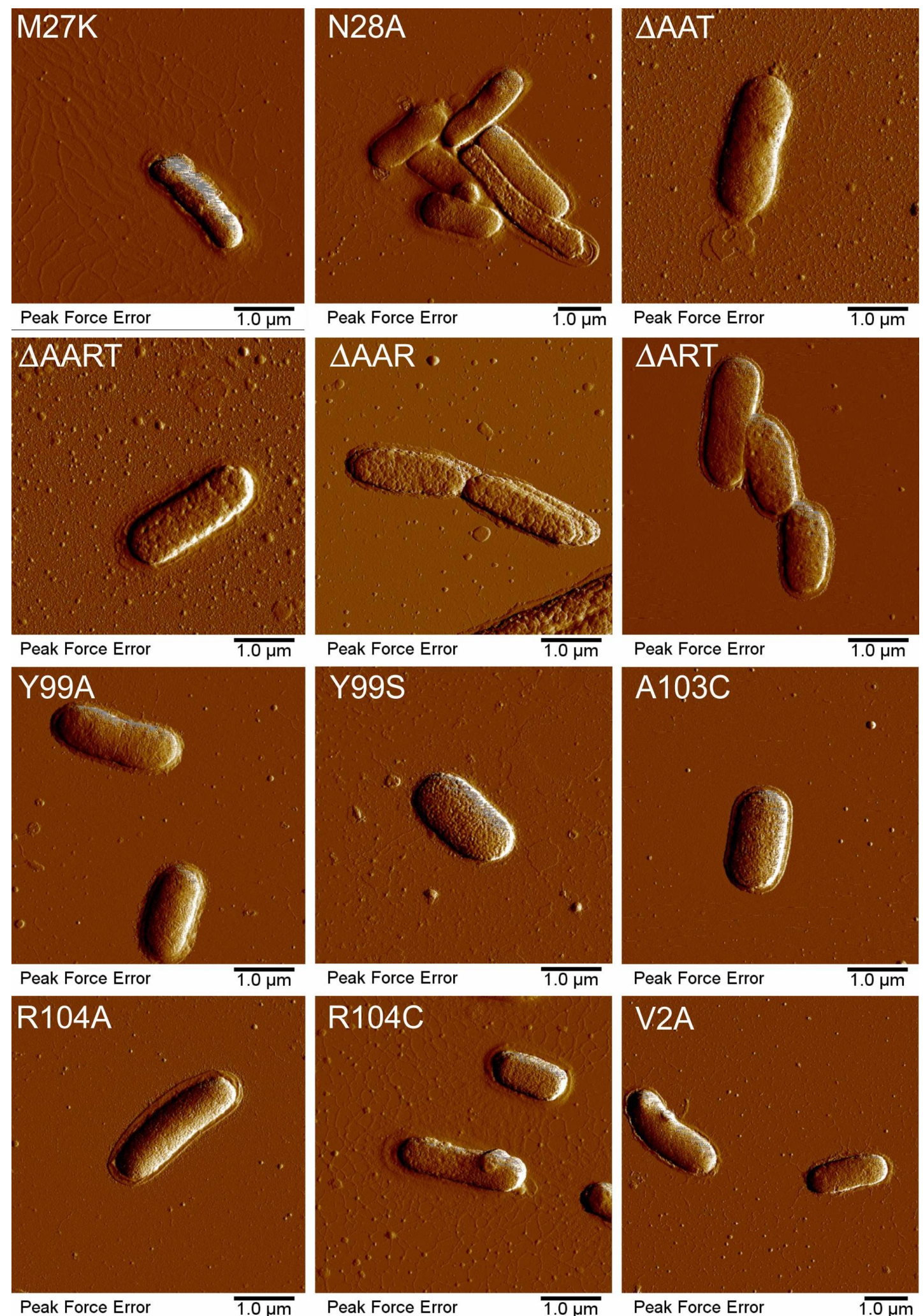

Peak Force Error

R104C

Peak Force Error

$1.0 \mu \mathrm{m}$

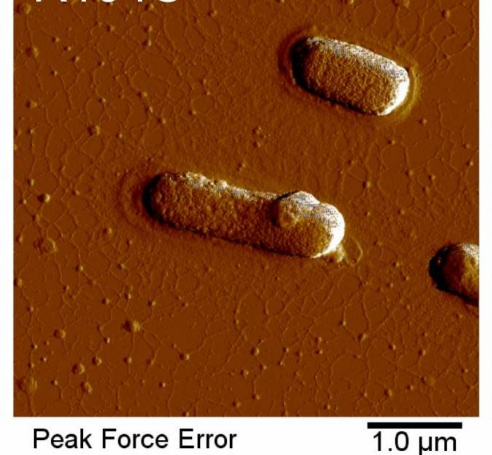

V2A

Continues on the next page 

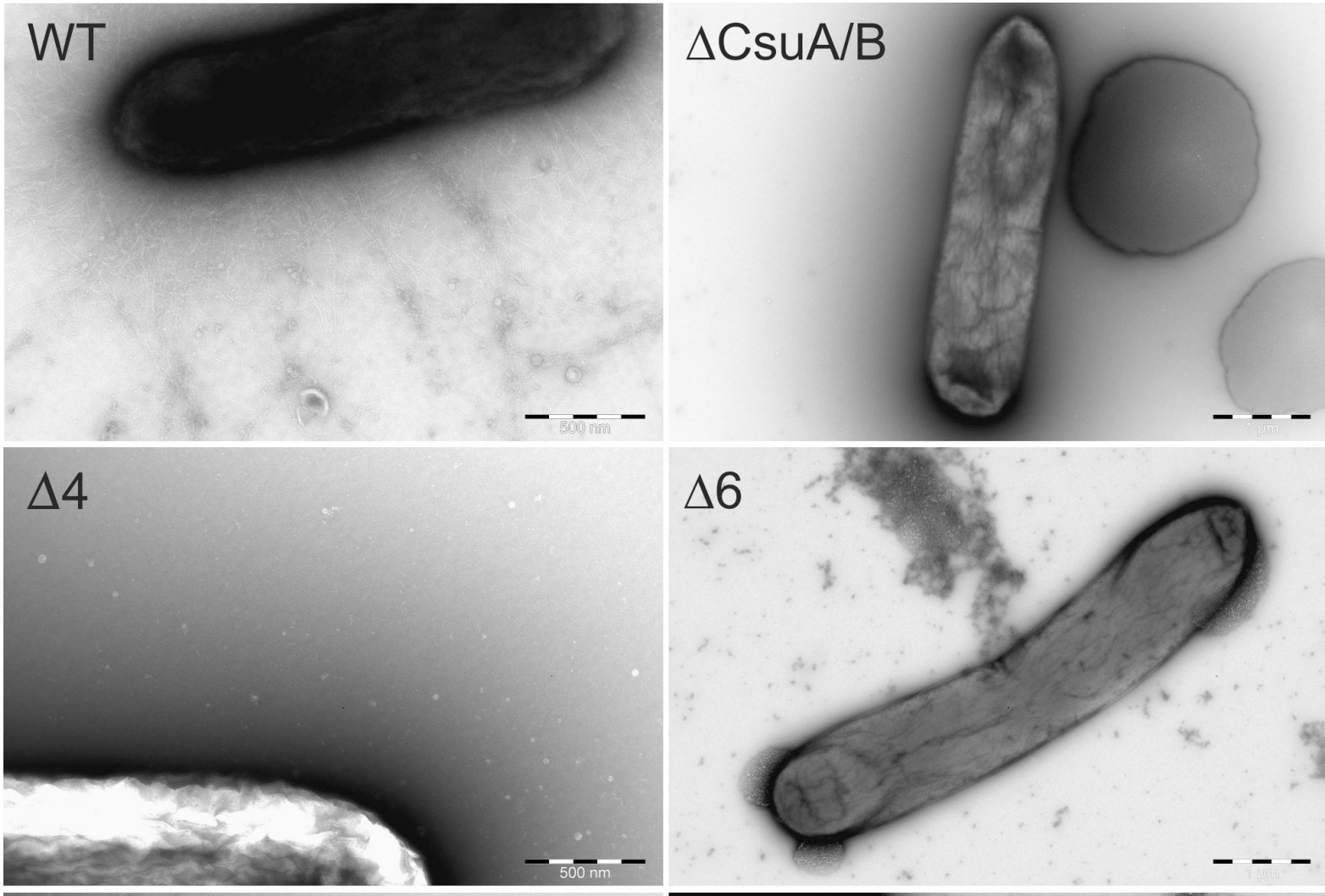

\section{$\Delta 6$}

\section{M27A}

\section{M27K}
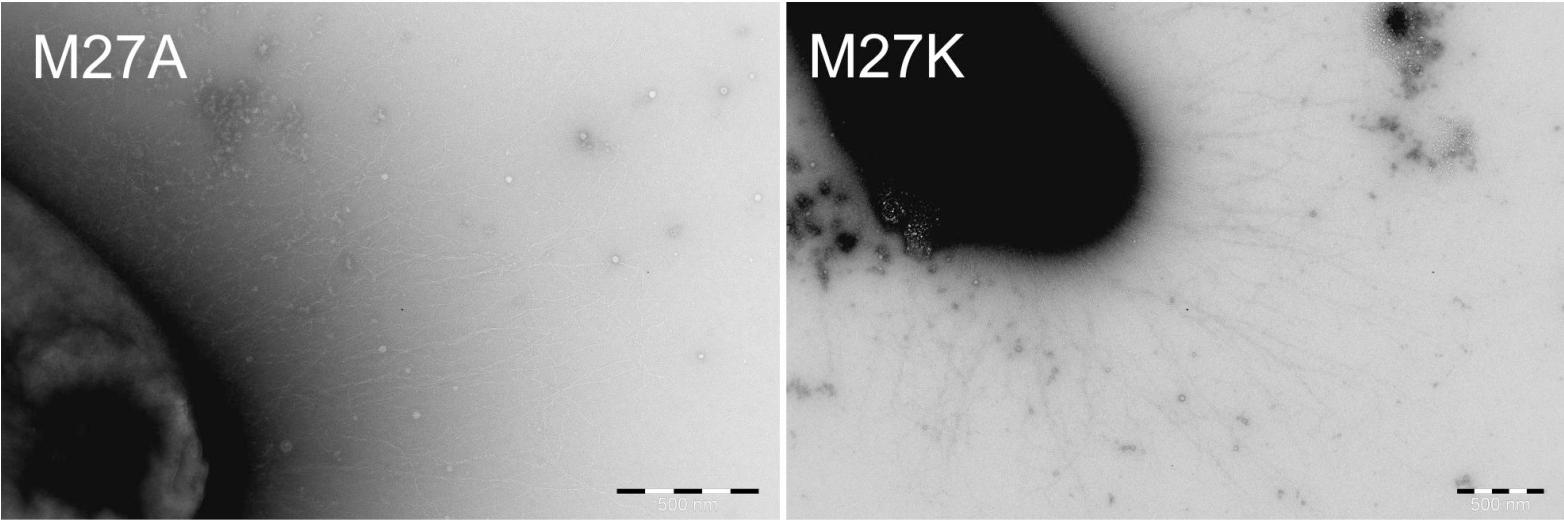

Extended Data Fig. 3 | Analysis of morphology of Csu pili on the bacterial cell surface. a, Atomic force microscopy images of E. coli expressing the wild type (WT) and mutant Csu pili. b, Transmission electron microscopy (TEM) micrographs of negative stained E. coli expressing the wild type (WT) and mutant Csu pili. Cells were cultured in LB medium in the presence of $0.02 \%$ arabinose. For the TEM imaging, specimens were stained with uranyl acetate. Mutations are explained in Extended Data Table 3. 


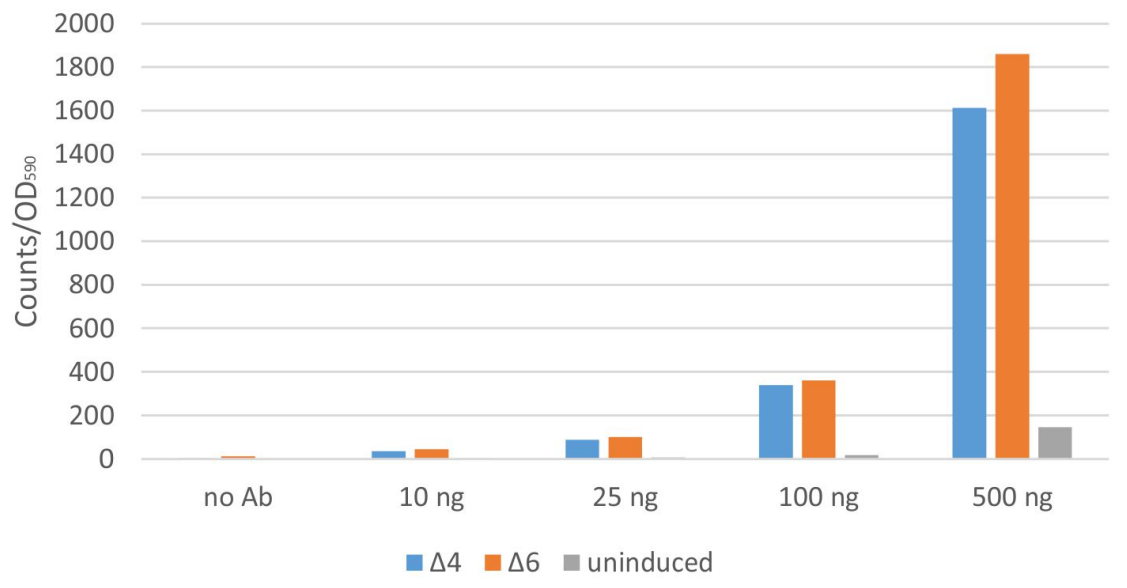

Extended Data Fig. 4 | CsuE is exposed on the cell surface despite of mutations preventing subunit clinching in the pilus rod. Recombinant E. coli harbouring the $C s u$ gene cluster, and expressing deletions $\Delta 4$ or $\Delta 6$ in the CsuA/B A'-A' 'hairpin (see Extended Data Table 3), were grown for two hours in the presence of arabinose to induce gene transcription. Uninduced cells were used as a negative control. Cells were divided in $4 \mathrm{ml}$ cultures and incubated for one hour further with different concentrations of polyclonal antibody raised against the N-terminal domain of $\mathrm{CsuE}$ subunit (anti-tip antibody or $\alpha \mathrm{E}_{\mathrm{N}}$ ) labelled with $\mathrm{Eu}^{3+-}$ chelate [N1-(4isothiocyanatobenzyl)diethylenetriamine-N1,N2,N3,N3-tetrakis(acetato)-europium(III)]. The bacteria were collected by centrifugation at $5000 \times \mathrm{g}$ and washed four times with phosphate buffered saline (PBS). The cells were resuspended in PBS, mixed 1:1 with the Europium Fluorescence Intensifier solution, and time-resolved $\mathrm{Eu}^{3+}$ fluorescence was measured in a 96well plate using a 1420 VICTOR Multilabel Counter. 

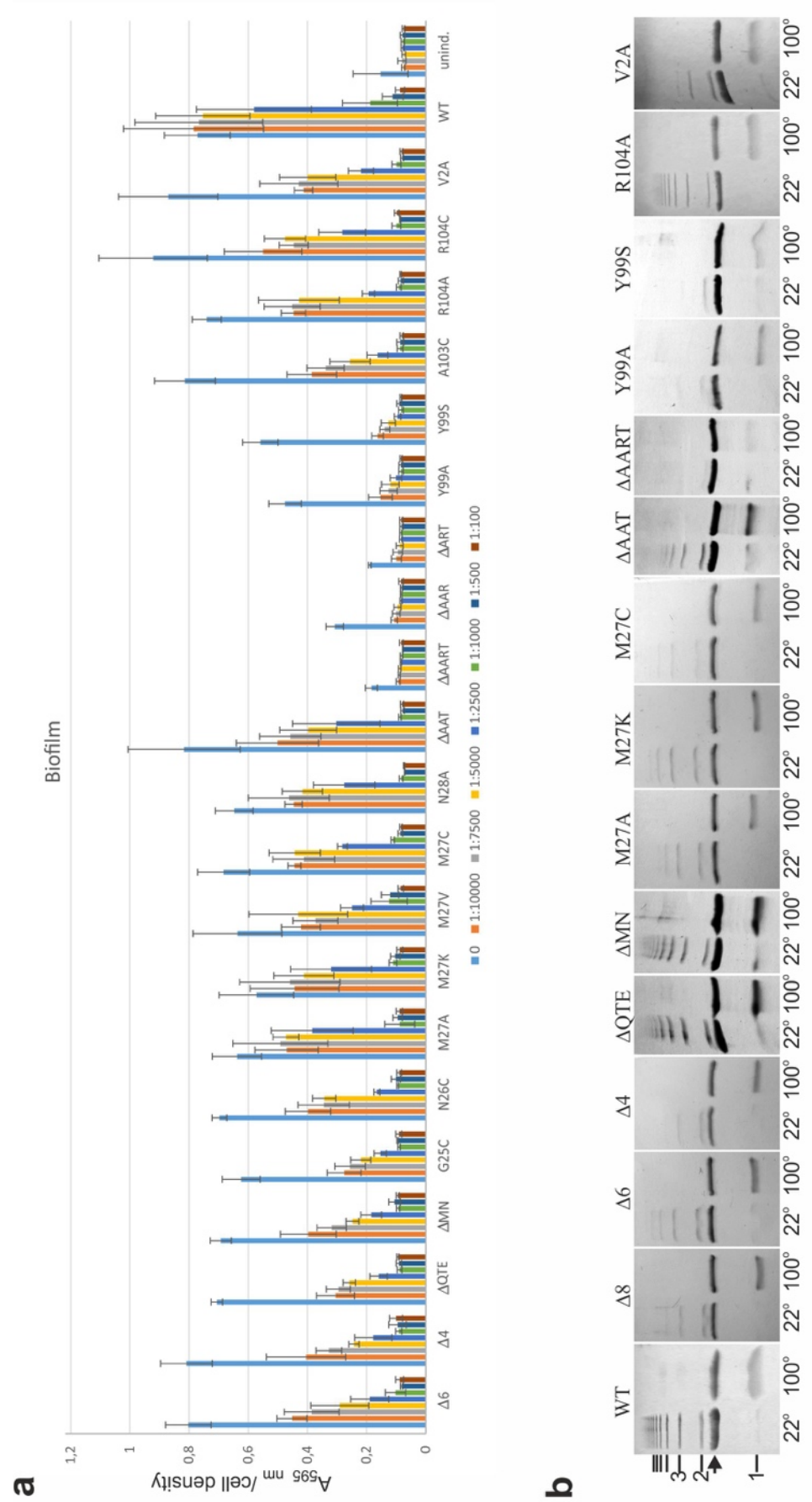

Extended Data Fig. 5 | Effects of mutations on Csu-mediated biofilm formation and usher-free polymerization of CsuA/B. a, Quantification of biofilms formed in polystyrene microtiter plates by $E$. coli harbouring the WT or the $\Delta 6$ mutant $c s u$ gene cluster at different concentrations of the anti-tip antibody. Uninduced cells were used as a negative control. Error bars represent standard deviations. $\mathbf{b}$, Effects of mutations on the ability of CsuA/B to form DSC-polymers. Wild type (WT) and mutant variants of CsuA/B were co-expressed with His6tagged CsuC in E. coli, co-purified from periplasmic extracts by $\mathrm{Ni}^{2+}$-affinity chromatography, and analysed by SDS-PAGE. Complexes were incubated at 22 or $100^{\circ} \mathrm{C}$ prior to the electrophoresis. Bars indicate positions of the melted CsuA/B monomer (1) and six short $\mathrm{CsuA} / \mathrm{B}$ polymers; the arrow shows position of the $\mathrm{CsuC}$ chaperone. Mutations are explained in Extended Data Table 3. 
a

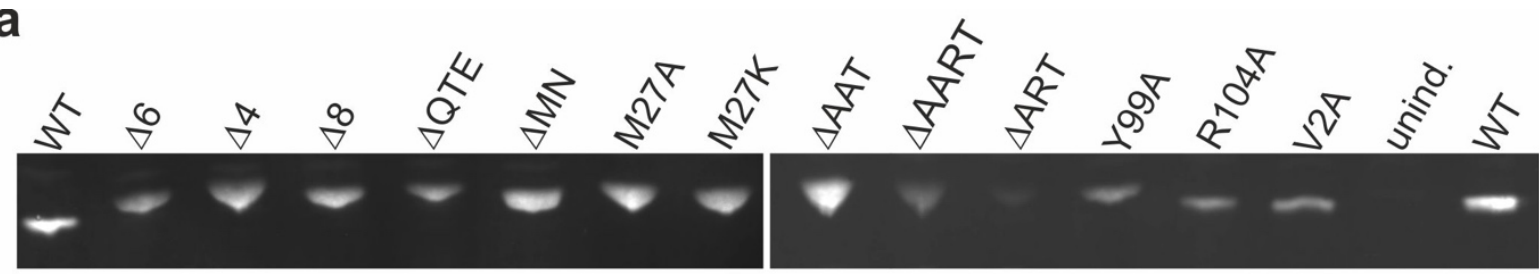

b

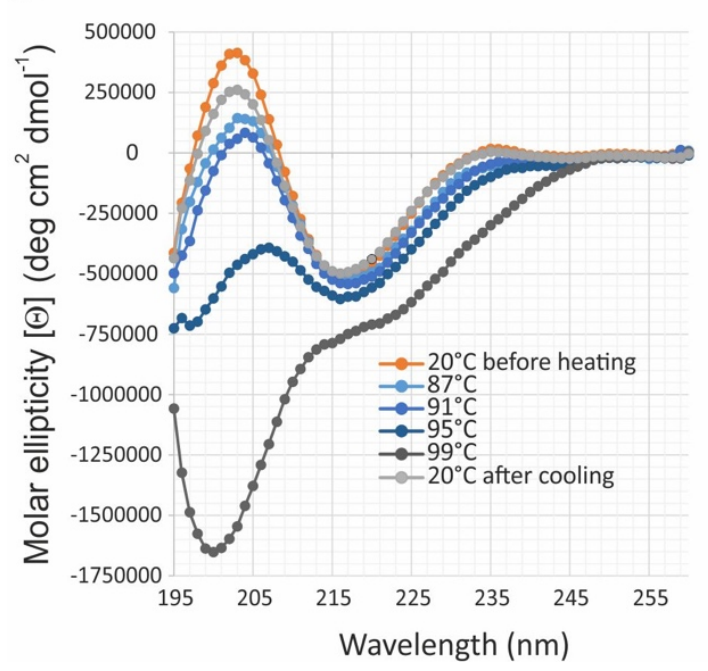

C

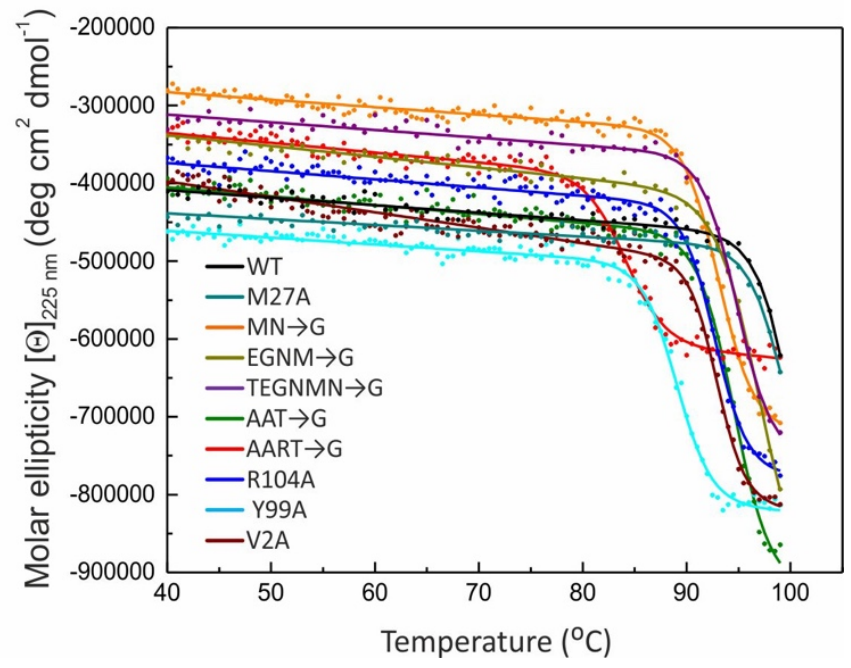

Extended Data Fig. 6 | Effect of mutations on the stability of donor strand complemented CsuA/B. a, Western blotting of periplasmic extracts of $E$. coli expressing WT and mutant CsuA/Bsc. Periplasmic extracts were obtained from equal number of cells. CsuA/Bsc was detected with rabbit polyclonal anti-CsuA/Bsc antibody and secondary IRDye 68RDconjugated anti-rabbit goat antibody as described in Procedures. b, CD spectra of WT CsuA/Bsc at different temperatures. $\mathbf{c}$, Temperature dependence of molar ellipticity of WT and substituted variants of CsuA/Bsc at $225 \mathrm{~nm}$. Melting temperatures generated from the data are listed in Extended Data Table 3. 


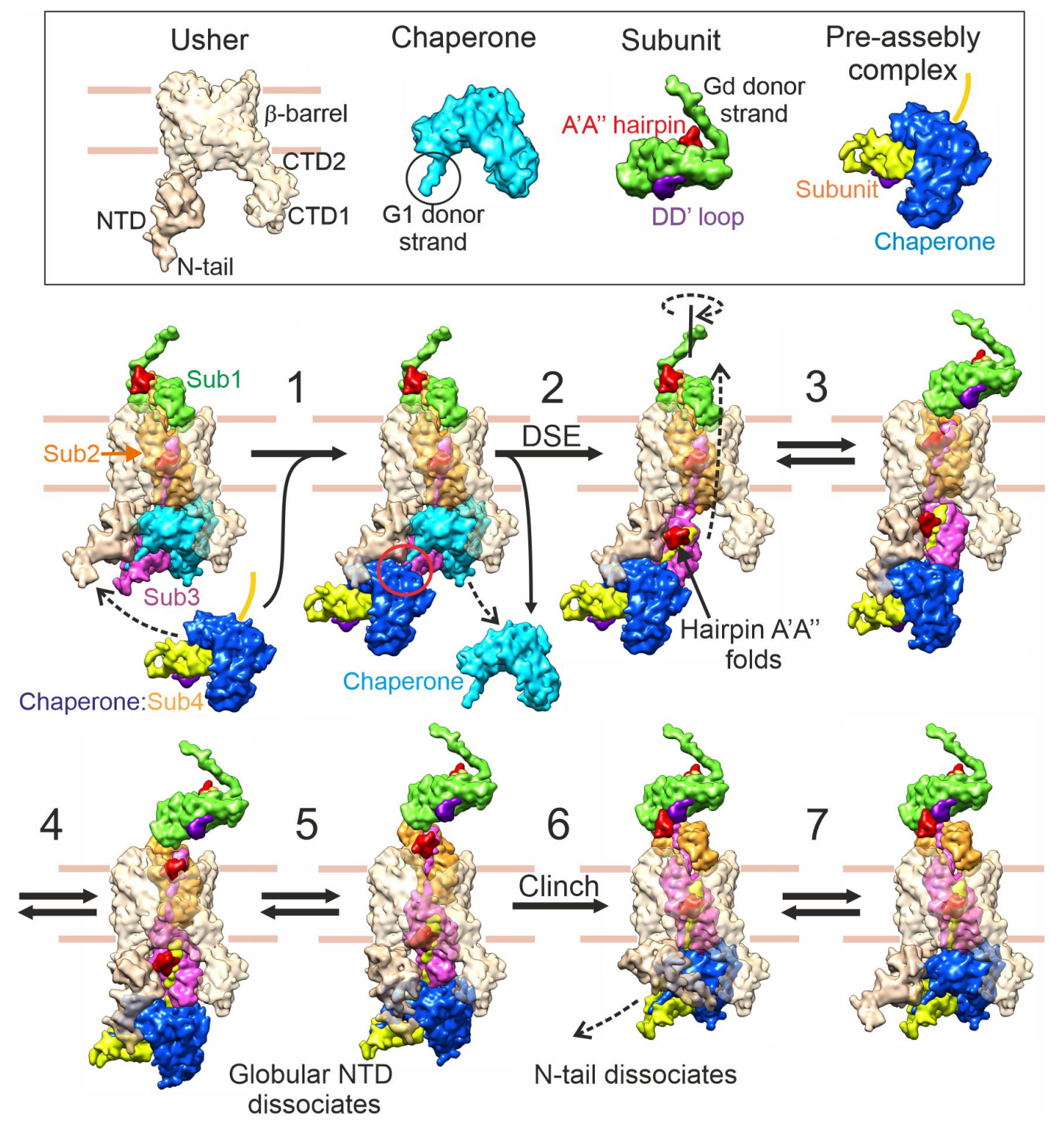

Extended Data Fig. 7 | Csu pilus assembly-secretion mechanism. The Csu pilus models were constructed based on the cryo-EM structure of the Csu-pilus rod (this article) and crystal structures of the self-complemented CsuA/B major subunit (PDB: 6FM5) ${ }^{27}$ and CsuC-CsuA/B chaperone-subunit complex (PDB: 5D6H) ${ }^{22}$. With no structure for the CsuD usher available, the models of the usher were based on the structures of the FimD usher from the classical CU pathway: the crystal structure (PDB: 3RFZ) ${ }^{24}$ and cryo-EM structures of conformers 1 and 2 (PDB codes 6E14 and 6E15, respectively, ${ }^{28}$. The Phyre2 protein fold recognition server 39 automatically modelled $92 \%$ residues of CsuD based on the structure of the entire FimD (conformer 1) with a confidence value of $100.0 \%$. Models of the N-terminal domain (NTD) of the usher at different steps of pilus secretion were produced based on the crystal structures of NTD bound to preassembly complexes (PDB codes 1ZE3 and 4B0M) ${ }^{23}{ }^{25}$ and cryo-EM structures of the FimD conformers. Steps:

1. The CsuC-CsuA/B chaperone-subunit preassembly complex with CsuA/B partially stabilized by the $\mathrm{G}_{1}$ donor strand of $\mathrm{CsuC}^{22}$ binds to the usher NTD, while a growing pilus represented here by a three CsuA/B subunit fragment (Sub1-Sub2-Sub3) in contact with the usher C-terminal domains CTD1 and CTD2. In classical CU systems, NTD mainly interacts with the chaperone using its $\mathrm{N}$-terminal tail (N-tail) and surface residues of the globular domain, which contribute 60 and $40 \% \%$ of the binding surface, respectively ${ }^{25}$. 
2. The Gd donor strand of the subunit in the preassembly complex (Sub4) replaces the $\mathrm{G}_{1}$ donor strand of the chaperone capping the base of the pilus in the zip-in-zip-out donor strand exchange (DSE) process ${ }^{14,16}$, linking Sub4 to the pilus. This also results in the complete folding of Sub3 and formation of the $\mathrm{A}^{\prime}-\mathrm{A}^{\prime \prime}$ and $\mathrm{B}-\mathrm{B}^{\prime}$ hairpins ${ }^{27}$. The former pilus-capping chaperone is released.

3-4. In a reversible process, the pilus translocates up the usher channel, rotating clockwise. Due to the presence of the $\mathrm{A}^{\prime} \mathrm{A}^{\prime \prime}-\mathrm{BB}^{\prime}$ twin hairpin, rotation within the usher is restricted to a relatively narrow path. To bring Sub2 $\mathrm{A}^{\prime}-\mathrm{A}^{\prime \prime}$ hairpin closer to the acceptor pocket on Sub1, secreted pilus or (most probably) the usher has to rotate as well (see Supplementary video 3).

5. Still in a reversible process, Sub2, emerging from the usher secretion channel, leans to the edge of the usher bringing Sub2 A'-A" hairpin closer to Sub1 DD' loop. The globular domain of NTD dissociates from CsuC as the angle between these proteins becomes suboptimal for the interaction, while the flexible N-tail remains bound as evident from the structure of FimD conformer $1^{28}$.

6. A clinch contact between Sub1 and Sub2 forms as the $\mathrm{A}^{\prime}-\mathrm{A}^{\prime \prime}$ hairpin of Sub2 and the Gd donor strand N-terminus of Sub3 bind to Sub1 from two sides, while the DD' loop covers the $\mathrm{A}^{\prime}-\mathrm{A}^{\prime \prime}$ hairpin from the side, locking its conformation with a second layer of interactions. The formation of the clinch is likely facilitated by narrowing down the number of pathways towards its formation: when subunits come close to one another, the $\mathrm{A}^{\prime}-\mathrm{A}^{\prime \prime}$ hairpin and Gd N-terminus prevent sideways rotations of Sub1 as shown on Fig. 2c. Once the clinch forms, Sub2 becomes part of the rigid pilus stalk and is unable to pull back into the usher channel. This mechanism prevents pilus backtracking, driving unidirectional secretion.

7. Upon the completion of the secretion cycle, the N-tail of the usher NTD reaches CTD2 and loses its affinity to the chaperone as suggested by Du et al. ${ }^{28}$. NTD is released to accept a new preassembly complex. 


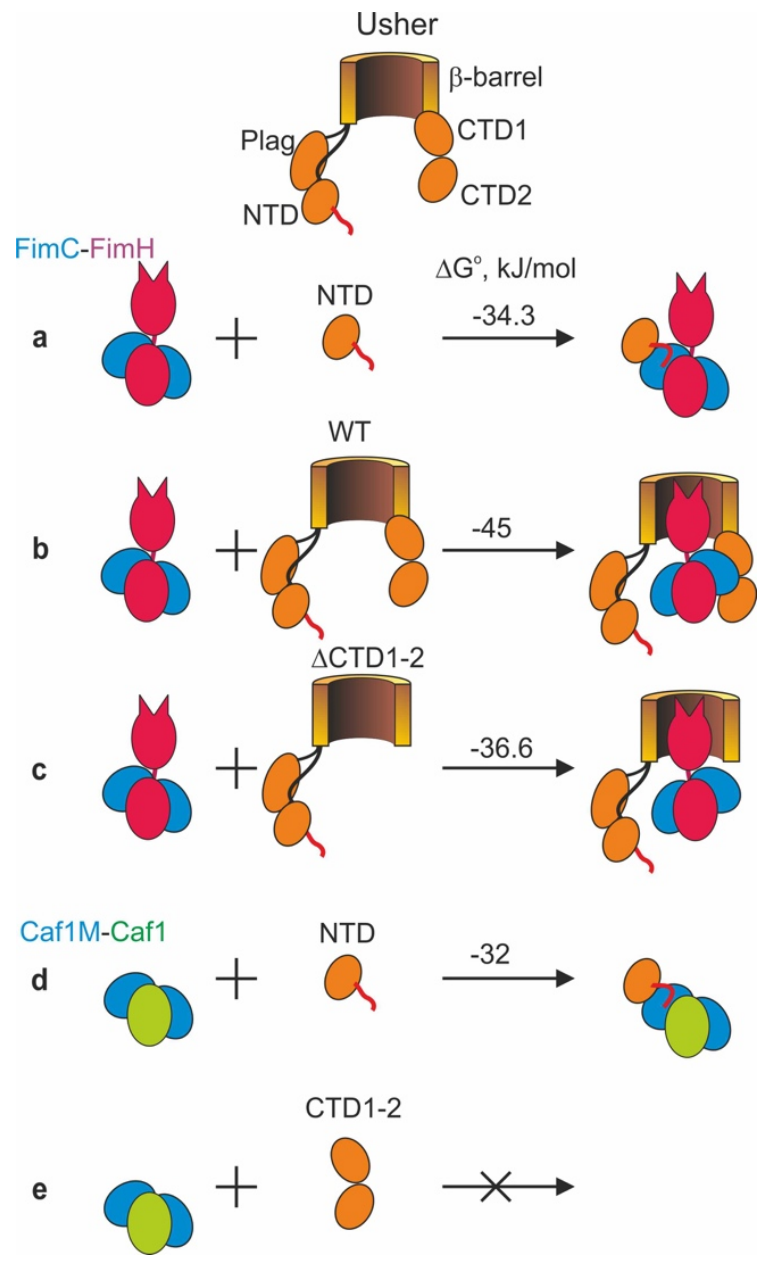

Extended Data Fig. 8 | Affinities of pre-assembly complexes to usher NTD and CTD1-2 in classical CUP systems. In classical CUPs, NTD mainly interacts with the chaperone using its $\mathrm{N}$-terminal tail (N-tail, wavy line), resulting in a rather strong binding affinity. Binding of the FimC-FimH chaperone-adhesin subunit complex to NTD of the FimD usher from the Type

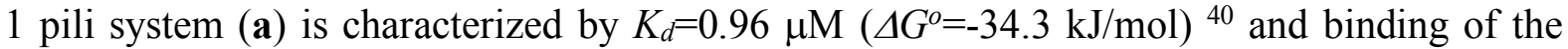
Caf1M-Caf1 chaperone-subunit complex to NTD of the Caf1A usher from the F1 antigen system (d) by $K_{d}=2.4 \mu \mathrm{M}\left(\Delta G^{o}=-32 \mathrm{~kJ} / \mathrm{mol}\right){ }^{25}$. However, no binding was detected beween the Caf1M-Caf1 chaperone-subunit or Caf1M-Caf1-Caf1 complexes with either single CTD2 ${ }^{41}$ or CTD1-2 constructs (e) (S. Roy and A. Zavialov, unpublished data). Although Werneburg et al. suggested the FimC-FimH complex has higher affinity for CTDs than NTD in the Type 1 pili system ${ }^{42}$, their excellent binding data seem to argue against this conclusion. The observed high affinity of the FimC-FimH complex to the usher $\left(K_{d}=12.5 \mathrm{nM}, \Delta G^{o}=-45 \mathrm{~kJ} / \mathrm{mol}\right)(\mathbf{b})$ is probably determined by the interactions of the lectin domain of FimH with the usher channel (c) rather than binding to CTDs since the deletion of CTDs in their study reduced the affinity only moderately ( 30 times, $\Delta G^{o}=-8.4 \mathrm{~kJ} / \mathrm{mol}$ ). Besides, the structural analysis of the Type 1 system 24,26 does not seem to reveal any particularly significant hydrophobic interactions between the base and CTDs that would corroborate the existence of tight binding between them, whereas the N-tail and preassembly complex form a considerable $\sim 600 \AA^{2}$ hydrophobic interface ${ }^{25}$. 


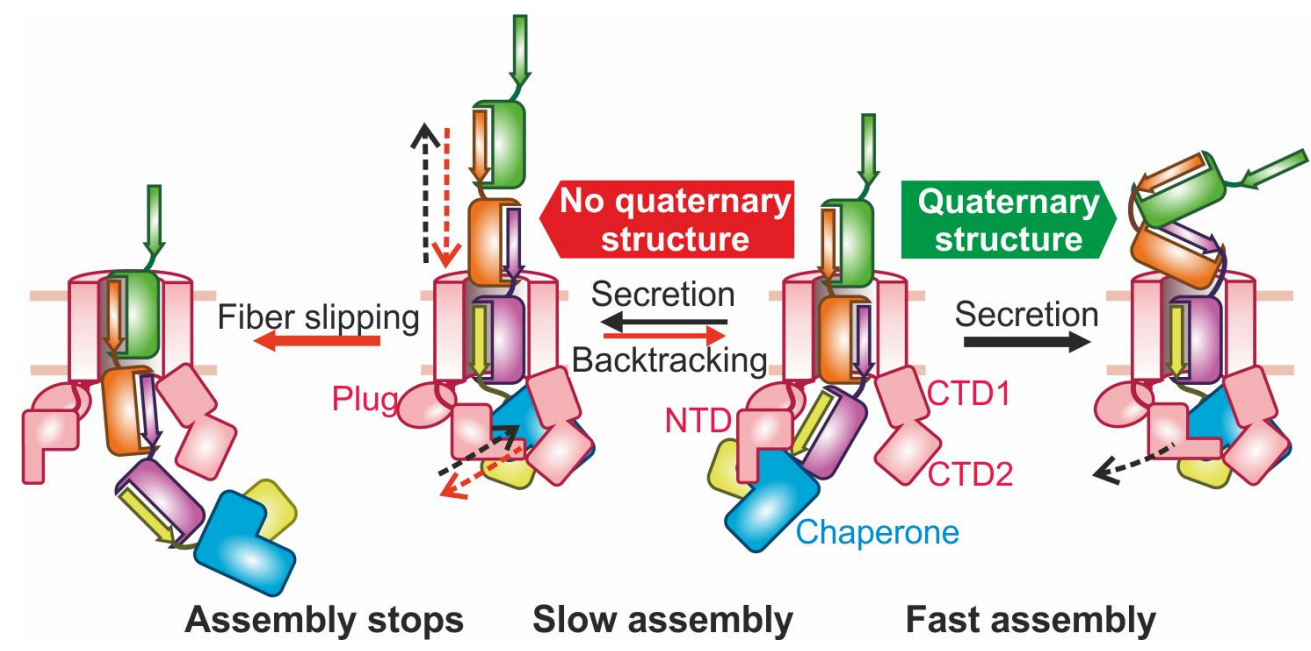

Extended Data Fig. 9 | Schematic representation of how pilus rod quaternary structure may prevent secretion backtracking and slipping of the fibre from the assembly platform. The pilus rod quaternary structure can potentially act as a ratchet-like mechanism preventing backtracking of the secretion step as well as highly hazardous accidents, in which the base slips away from the usher after its release from NTD, permanently jamming assembly. See also Supplementary Video 3. 
a

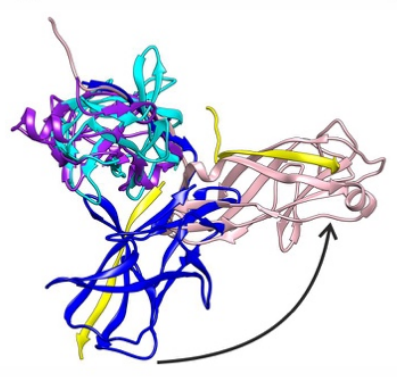

b
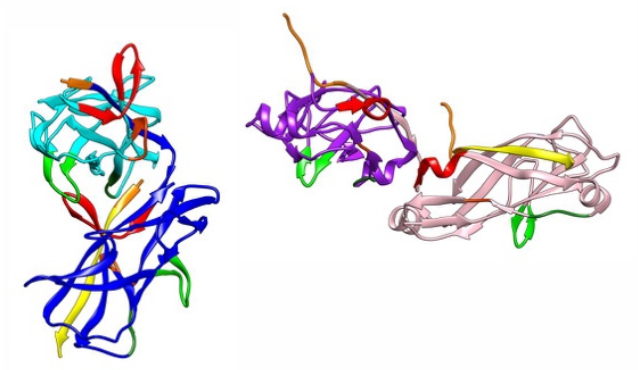

C

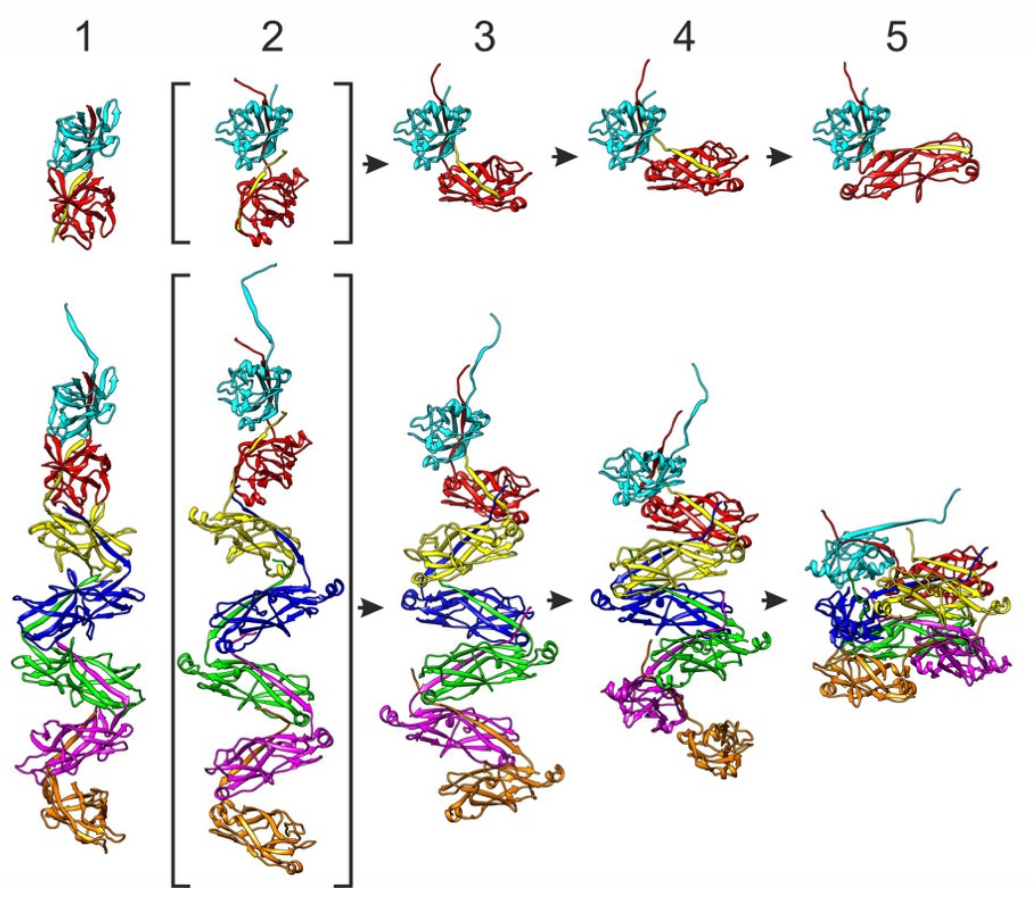

Extended Data Fig. 10 | Angles between subunits in the zigzag and helical tube pilus rods and probable evolutionary pathway between the two architectures. a, Fragments of Csu and Pap pilus rods consisting of two adjacent subunits $(\mathrm{N}$ and $\mathrm{N}+1)$ and the donor strand from the third subunit $\left(\mathrm{Gd}^{\mathrm{N}+2}\right)$ were superimposed over $\mathrm{C} \alpha$ atoms of the first subunits. $\mathrm{CsuA} / \mathrm{B}^{\mathrm{N}}$ and $\mathrm{CsuA} / \mathrm{B}^{\mathrm{N}+1}$ are painted cyan and blue, while $\mathrm{Pap}^{\mathrm{N}}$ and $\mathrm{PapA}^{\mathrm{N}+1}$ are painted violet and pink, respectively. $\mathrm{Gd}^{\mathrm{N}+2}$ is painted yellow. A change from the zigzag architecture to tube-like helix requires a rotation of the second subunit by approximately 90 degrees as indicated by an arrow. b, Same fragments as in (A) shown separately. The $\mathrm{A}^{\prime}-\mathrm{A}^{\prime \prime}$ hairpin, N-terminal part of the Gd strand, DD' loop, and other regions involved in the clinch contact between subunits in the Csu rod are painted red, orange, green, and dark green respectively. Corresponding regions in the PapA subunits are painted the same colours. c, Predicted evolutionary pathway from a zigzag to a helical tube architecture. The images show adjacent subunits as in (A) to illustrate changes in the inter-subunit angle (upper images) and seven-subunit pilus rod fragments to illustrate changes in the rod architecture (lower images). (1) The Csu pilus, a member of the archaic CU fimbriae. The $\mathrm{A}^{\prime}-\mathrm{A}^{\prime \prime}$ hairpin locks the angle between subunits. (2) A hypothetical model obtained by superimposing PapA subunits over CsuA/B subunits in the Csu rod. This hypothetical structure suggests that a deletion (or shortening) of the $\mathrm{A}^{\prime}-\mathrm{A}^{\prime \prime}$ hairpin may have enabled rotation of subunits relative to each other to form a more compact helical packing. At the same time, a longer $\mathrm{N}$-terminal tail may have evolved to stabilize the inter-subunit contact in the absence of the $\mathrm{A}^{\prime}-\mathrm{A}^{\prime \prime}$ hairpin. (3) A hypothetical early intermediate. The transition of $\mathrm{A}^{\prime}-$ 
$\mathrm{A}^{\prime \prime}$ hairpin (as in CsuA/B) into an $\alpha$-helix (as in PapA) may have enabled change in the angle between subunits, while the N-terminal tail of Gd donor strand may have helped maintain stability. (4) A hypothetical late intermediate. Further change in the angle may have enabled the formation of various open spiral architectures still lacking connections between the subunit layers. (5) Pap pilus, a member of the classical CU fimbriae. The final step in the evolution was the formation of interactions between subunit layers. Most structures required to maintain the stability of open spiral structures have become unnecessary and disappeared. 
Extended Data Table 1 | Cryo-EM data collection, helical reconstruction, and validation

\begin{tabular}{ll}
\hline Data collection & \\
\hline Magnification & 105,000 \\
Voltage $(\mathrm{kV})$ & 300 \\
Electron exposure $\left(\mathrm{e}^{-} / \AA^{2}\right)$ & 60 \\
Defocus range $(\mu \mathrm{m})$ & -1.0 to -3.0 \\
Pixel size $(\AA)$ & 0.433 \\
\hline
\end{tabular}

\begin{tabular}{ll}
\hline Helical reconstruction & \\
\hline Box size (pix) & 400 \\
Inter-box distance (pix) & 60 \\
Number of asymmetric units/box & 1 \\
Maximum curvature $(\AA)$ & 0.4 \\
Number of picked segments & 480,064 \\
Number of segments used for 3D refinement & 255,833 \\
Helical rise $(\AA)$ & 27.97 \\
Helical twist $\left({ }^{\circ}\right)$ & -152.8 \\
Map sharpening B-factor $\left(\AA^{2}\right)$ & -144.18 \\
Final map resolution $(\AA)$ at FSC 0.143 & 3.42
\end{tabular}

Model building, refinement and validation

\begin{tabular}{ll}
\hline PDB code used for model building & 6 FM5 \\
Model composition & \\
Non-hydrogen atoms & 3387 \\
Amino acid residues & 465 \\
R.m.s. deviations & \\
Bond lengths $(\AA)$ & 0.007 \\
Bond angles ( $\left.{ }^{\circ}\right)$ & 1.388 \\
MolProbity score & 1.11 \\
All-atom clash score & 3.15 \\
Ramachandran plot & \\
Favoured (\%) & 98.12 \\
Allowed $(\%)$ & 1.75 \\
Disallowed (\%) & 0 \\
Bad rotamers (\%) & 0 \\
Model-to-map fit & \\
CC_mask & 0.68 \\
CC_volume & 0.70 \\
CC_peak & 0.76 \\
CC_box & 0.75 \\
\hline
\end{tabular}


Extended Data Table 2 | Oligonucleotides

\begin{tabular}{|c|c|c|}
\hline Mutation ${ }^{a}$ & Forward & Reverse \\
\hline$\Delta$ CsuE & TCATGGCAAAGATACCTCGTGA & TAAAAGCTGTTTTATATAGGAGATAAAAAG \\
\hline$\Delta \mathrm{CsuA} / \mathrm{B}$ & GAАтTCCGTTAАTTCCTCCTGTTAG & GAGTAGCAGGTTTGCTCAAATATG \\
\hline$\Delta 8$ & САСTACCACCTACAGTACAGCC & GATTTGGTACTTTAAATTTTGGTAAAACTTC \\
\hline$\Delta 6$ & CTTGACTACCACCTACAGTACAG & GTAAGTTTGGTACTTTAAATTTTGGTAAAAC \\
\hline$\Delta 4$ & CAGTTTGACTACCACCTACAGTAC & GTAACAAGTTTGGTACTTTAAATTTTGGTAAAAC \\
\hline$\triangle Q T E$ & CACTACCACCTACAGTACAGCC & GTGGAAATATGAACAAGTTTGGTACTTTAAATTTTG \\
\hline$\Delta \mathrm{MN}$ & сAтTTCстTCAGTTTGACTACCAC & GTAAGTTTGGTACTTTAAATTTTGGTAAAAC \\
\hline G25C & САTTCAGTTTGACTACCACCTACAGTAC & TAATATGAACAAGTTTGGTACTTTAAATTTTGG \\
\hline N26C & TGACTACCACCTACAGTAC & $\begin{array}{l}\text { AACTGAAGGATGTATGAACAAGTTTGGTACTTTAA } \\
\text { ATTTTG }\end{array}$ \\
\hline M27A & GСАTTTCСTTCAGTTTGACTAССАССТАС & GAACAAGTTTGGTACTTTAAATTTTGGTAAAAC \\
\hline M27K & TTATTTCCTTCAGTTTGACTAССАССТАС & GAACAAGTTTGGTACTTTAAATTTTGGTAAAAC \\
\hline M27V & GTTTGACTACCACCTACAG & TGAAGGAAATGTGAACAAGTTTGGTAC \\
\hline M27C & GTTTGACTACCACCTACAGTACAG & TGAAGGAAATTGTAACAAGTTTGGTAC \\
\hline N28A & TCAGTTTGACTACCACCTACAGTACAG & AgGAAATATGGCCAAGTTTGgTACTTTAAATTTTG \\
\hline$\triangle \mathrm{AAT}$ & CTGAAGCAACTTCAGCTGTTAATAC & GTGGTGGCAATATTTCTGTGACTTG \\
\hline$\triangle \mathrm{AART}$ & САTCACGATAAACGTTATATGCAACTAC & GTAACCTTTATGTTGTAAАCCAАCCAC \\
\hline$\triangle \mathrm{AART}$ & CATCACGATAAACGTTATATGCAACTAC & GTACAAACCTTTATGTTGTAAACCAACCAC \\
\hline$\triangle \mathrm{ART}$ & CTCCAGCATCACGATAAACGTTATATG & GTAACCTTTATGTTGTAAACCAACCAC \\
\hline Y99A & CAACGTTATATGCAACTACATCAGCAG & CTCGTGATGCTGCACGTACAAAC \\
\hline Y99S & \multicolumn{2}{|c|}{ This substitution appeared by accident with oligos designed to produce Y99A } \\
\hline A103 & TAAACGTTATATGCAACTACATCAGCAGAAGC: & TCGTGATGCTTGTCGTACAAACCTTTATGTTG \\
\hline R104A & CTGCAGCATCACGATAAACGTTATATG & СТАСАAАССТTTATGTTGTAAACCAАCСАC \\
\hline R104C & CGATAAACGTTATATGCAACTAC & TGATGCTGCATGTACAAACCT \\
\hline V2A & AGTATTTACCGCATAACCAGCAAC & CAAGCAGCTGCTACTGGTCAG \\
\hline $\begin{array}{l}\text { V2A for } \\
\text { CsuA/Bsc }\end{array}$ & $\begin{array}{l}\text { CATTTTGTTTGTTATCGAAATTTACAGTGAC } \\
\text { TAATAG }\end{array}$ & GAGGTGCTGCTACTGGTCAGG \\
\hline
\end{tabular}

${ }^{a}$ Mutations are explained in Extended Data Table 3 


\begin{tabular}{|c|c|c|c|c|c|c|c|c|}
\hline \multirow[t]{3}{*}{ Name } & \multirow[t]{3}{*}{ Mutation } & \multirow[t]{3}{*}{ Site } & \multicolumn{3}{|c|}{ Phenotype } & \multirow{3}{*}{$\begin{array}{l}\text { Usher-free } \\
\text { assembly }^{c}\end{array}$} & \multicolumn{2}{|c|}{ Stability of CsuA/Bsc } \\
\hline & & & \multirow{2}{*}{ Pili $^{a}$} & \multicolumn{2}{|c|}{ Biofilm $^{\text {b }}$} & & \multirow{2}{*}{$\begin{array}{c}\text { Expression } \\
\text { level }^{\mathrm{d}}\end{array}$} & \multirow{2}{*}{$T_{m},{ }^{o} C^{e}$} \\
\hline & & & & No inhibition & $\alpha E_{N}, 1: 2500$ & & & \\
\hline Wild type & & & ++++ & $1.0 \pm 0.15$ & $0,81 \pm 0,27$ & ++++ & ++++ & $\sim 100$ \\
\hline Uninduced & & & - & - & - & & & \\
\hline$\Delta$ CsuE & $\Delta \mathrm{CsuE}$ & & - & - & - & & & \\
\hline$\Delta \mathrm{CsuA} / \mathrm{B}$ & $\Delta \mathrm{CsuA} / \mathrm{B}$ & & - & - & - & & & \\
\hline$\Delta 8$ & QTEGNMNK $\rightarrow$ G & A'A" hairpin & NT & NT & NT & ++++ & ++++ & NT \\
\hline$\Delta 6$ & $\mathrm{TEGNMN} \rightarrow \mathrm{G}$ & A'A" hairpin & - & $1.05 \pm 0.11$ & $0.18 \pm 0.09$ & ++++ & ++++ & $94.8 \pm 0.2$ \\
\hline$\Delta 4$ & $\mathrm{EGNM} \rightarrow \mathrm{G}$ & A'A"' hairpin & - & $1.06 \pm 0.12$ & $0.16 \pm 0.09$ & ++++ & ++++ & $97.0 \pm 0.4$ \\
\hline$\triangle Q T E$ & $\mathrm{QTE} \rightarrow \mathrm{G}$ & A'A" hairpin & - & $0.89 \pm 0.03$ & $0.13 \pm 0.04$ & ++++ & ++++ & NT \\
\hline$\Delta \mathrm{MN}$ & $\mathrm{MN} \rightarrow \mathrm{G}$ & A'A" hairpin & $-/+$ & $0.87 \pm 0.05$ & $0.17 \pm 0.05$ & ++++ & ++++ & $93.1 \pm 0.1$ \\
\hline G25C & G25C & A'A"' hairpin & - & $0.76 \pm 0.09$ & $0.12 \pm 0.03$ & NT & NT & NT \\
\hline N26C & N26C & A'A"' hairpin & - & $0.88 \pm 0.035$ & $0.14 \pm 0.01$ & NT & NT & NT \\
\hline M27A/K/V & $\mathrm{M} 27 \rightarrow \mathrm{A}$ or $\mathrm{K}$ or $\mathrm{V}$ & A'A" hairpin & ++++ & $0.78 \pm 0.12_{\mathrm{A}}$ & $0.49 \pm 0.20_{A}$ & ++++ & ++++ & $99 \pm 2 \mathrm{~A}$ \\
\hline N28A & $\mathrm{N} 28 \mathrm{~A}$ & A'A" hairpin & + & $0.80 \pm 0.09$ & $0.32 \pm 0.15$ & NT & NT & NT \\
\hline$\triangle \mathrm{AAT}$ & $\mathrm{AAT} \rightarrow \mathrm{G}$ & BB' hairpin & $-/+$ & $1.07 \pm 0.27$ & $0.36 \pm 0.21$ & ++++ & ++++ & $94.6 \pm 0.1$ \\
\hline$\triangle \mathrm{AART}$ & $\mathrm{AART} \rightarrow \mathrm{G}$ & DD' loop & - & $0.05 \pm 0.03$ & $0.009 \pm 0.003$ & - & ++ & $84.1 \pm 0.1$ \\
\hline$\triangle \mathrm{AAR}, \Delta \mathrm{ART}$ & $\mathrm{AAR}, \mathrm{ART} \rightarrow \mathrm{GG}$ & DD' loop & - & $0.25 \pm 0.04_{\mathrm{AAR}}$ & $0.01 \pm 0.005_{\mathrm{AAR}}$ & - & $+{ }_{\mathrm{AAR}}$ & NT \\
\hline Y99A/S & $Y 99 \rightarrow A$ or $S$ & DD' loop & - & $0.53 \pm 0.08_{A}$ & $0.036 \pm 0.03_{A}$ & +++ & +++ & $89.0 \pm 0.1_{\mathrm{A}}$ \\
\hline A103C & $\mathrm{A} 103 \mathrm{C}$ & DD' loop & - & $1.07 \pm 0.15$ & $0.14 \pm 0.05$ & NT & NT & NT \\
\hline R104A & R104A & DD' loop & $-/+$ & $0.95 \pm 0.07$ & $0.19 \pm 0.03$ & ++++ & +++ & $92.7 \pm 0.1$ \\
\hline R104C & R104C & DD' loop & ++ & $1.24 \pm 0.26$ & $0.33 \pm 0.11$ & NT & NT & NT \\
\hline V2A & V2A & $\mathrm{N}$-terminus & + & $1.16 \pm 0.24$ & $0.23 \pm 0.06$ & ++++ & +++ & $92.9 \pm 0.1$ \\
\hline
\end{tabular}

${ }^{a}$ Atomic force microscopy (AFM) (Extended Data Fig. 3a) and negative stain transmission electron microscopy (TEM) imaging of bacterial cells (Fig. 4c and Extended Data Fig. 3b). The number of pluses indicates the amount of pili: ++++ , amount similar to that of the wild type (WT); + to +++, a reduced number of WT-like pili; -/+, pilus-like structures observed on some bacterial cells but not others; -, no pili observed. NT, not tested.

${ }^{b}$ Csu-mediated biofilms on plastics with or without anti-tip $\left(\alpha E_{N}\right)$ antibody. The density of uninduced cells was subtracted from the biofilm cell density data (Fig. $4 \mathrm{~d}$ and Extended Data Fig. 5a) and normalized to the biofilm density of WT cells in the absence of $\alpha \mathrm{E}_{\mathrm{N}}$. The result of biofilm inhibition by $\alpha \mathrm{E}_{\mathrm{N}}$ diluted 1:2500 times is shown (see Fig. 5a for the full set of data).

${ }^{c}$ CsuC-assisted assembly of CsuA/B (Fig. 4b and Extended Data Fig. 5b). ++++, WT-like polymerization efficiency; +++, most subunits form DSC-polymers; -, not detected due to poor yield.

${ }^{\mathrm{d}}$ Relative level of WT and mutant CsuA/Bsc in the E. coli periplasm (Fig. 4a and Extended Data Fig. 6a).

e Melting temperature $\left(T_{m}\right)$ of WT and mutant CsuA/Bsc obtained by measuring circular dichroism at different temperatures (Extended Data Fig. 6b, c). 


\section{Supplementary Files}

This is a list of supplementary files associated with this preprint. Click to download.

- structuref.mp4

- jaws.mp4

- assembly11MB.mp4 